\title{
Démographie médicale et carrières des médecins généralistes : les inégalités entre générations
}

\author{
Brigitte Dormont* et Anne-Laure Samson**
}

En France, l'offre de soins ambulatoires est régulée depuis 1971 par le numerus clausus, qui fixe le nombre d'étudiants admis en deuxième année de médecine. Fixé initialement à 8588 places, il n'a vraiment diminué qu'à partir de 1978, jusqu'à atteindre 3500 places en 1993. L'arrivée des cohortes nombreuses du baby-boom et la faiblesse des restrictions initiales ont permis l'installation de générations nombreuses de médecins débutants. Ce n'est qu'à partir de 1987 (soit neuf ans après 1978, du fait de la durée des études médicales) que l'on observe un impact du numerus clausus sur le nombre de médecins débutants. Un panel représentatif des généralistes du secteur 1 sur la période 1983-2004 permet d'analyser les déterminants de leurs honoraires et l'impact des fluctuations du numerus clausus sur leurs carrières.

La localisation et les revalorisations tarifaires ont un impact considérable sur les honoraires. Les profils de carrières des médecins diffèrent fortement de ceux des salariés : toutes choses égales par ailleurs, leurs honoraires progressent rapidement en début de carrière pour diminuer en moyenne dès la douzième année d'expérience. Tout se passe comme si les médecins préféraient concentrer leur effort au début de leur expérience professionnelle pour alléger ensuite leur charge de travail.

Les honoraires des médecins dépendent fortement de la situation de la démographie médicale lors de leur installation. L'écart entre les honoraires permanents des différentes cohortes peut atteindre $20 \%$, toutes choses égales par ailleurs. Les cohortes installées dans les années 1980 subissent les impacts conjoints du baby-boom et d'un numerus clausus élevé : elles perçoivent les honoraires les plus faibles. La diminution du numerus clausus a permis d'améliorer la situation des cohortes ultérieures. Une comparaison des distributions d'honoraires en termes de dominance stochastique montre que les écarts liés à l'hétérogénéité non observée ne compensent pas les différences moyennes entre cohortes.

\footnotetext{
* Greqam (CNRS), Université Paris Dauphine (LEDA-Legos) et IEMS, Lausanne. E-mail : brigitte.dormont@dauphine.fr ** EconomiX, Université Paris 10-Nanterre. E-mail : anne-laure.samson@u-paris10.fr

Cette étude a été réalisée dans le cadre d'une convention de recherche entre l'université Paris Dauphine et la Direction de la recherche, des études, de l'évaluation et des statistiques (Drees) du Ministère de la Santé, de la Jeunesse, des Sports et de la vie associative. Les auteurs remercient Nicolas Pistolesi pour ses programmes informatiques sur les tests de dominance stochastique. Ils remercient aussi, pour leurs remarques et suggestions, trois rapporteurs anonymes de la revue, ainsi que Yann Bourgueil, Julien Pouget, Sandy Tubeuf, les participants des séminaire 3S (Drees), IEMS et PSE, ceux des 6èmes journées Louis-André Gérard-Varet (Marseille, Juin 2007), ceux du $16^{\text {th }}$ Workshop on Health Economics and Econometrics (Bergen, Septembre 2007) et ceux des 56 $6^{\text {emes }}$ Journées de l'AFSE (Paris, Septembre 2007).
} 
$\mathbf{E}$ n France, l'organisation de la médecine ambulatoire se caractérise par la coexistence d'un financement sur prélèvements obligatoires et d'une grande liberté des acteurs au niveau décentralisé : pour les patients, la liberté de choix du médecin est totale (1). Pour les médecins, la liberté d'installation est entière : les mesures visant à améliorer la répartition géographique de l'offre de soins ambulatoires ont toujours privilégié l'approche incitative. À cela s'ajoute un système de paiement à l'acte, qui relie étroitement les revenus du médecin au nombre d'actes délivrés.

On conçoit dans ces conditions les difficultés connues pour le pilotage des dépenses en médecine ambulatoire. En dehors des difficultés institutionnelles liées à la répartition des pouvoirs de décision entre différentes instances administratives (Cour des Comptes, 2007), l'organisation même du système rend difficile la maîtrise de la progression des dépenses et la recherche de leur efficacité.

En matière de régulation de l'offre de soins ambulatoires, le seul outil véritablement utilisé est le numerus clausus : introduit en 1971, il fixe le nombre de places en deuxième année des études de médecine. Nombre de débats se focalisent sur son niveau le plus approprié. Fixé initialement à 8588 , il n'a véritablement diminué qu'à partir de 1978, jusqu'à atteindre en 1993 un plancher de 3500 places. La timidité des restrictions initiales a d'abord permis une augmentation spectaculaire du nombre de médecins libéraux, lequel a pratiquement doublé en trente ans (HCAAM, 2007). Du fait de la durée des études médicales, l'impact du numerus clausus sur les effectifs de médecins débutants n'a pu être observé qu'à partir de 1987 (2). L'amorce d'une diminution de la densité médicale n'apparaît qu'au début des années 2000 (Bessière et $a l ., 2004)$. Le spectre d'une pénurie de médecins est maintenant régulièrement agité pour obtenir une augmentation du numerus clausus. Pour 2008, celui-ci est relevé à 7300 places. Cette mesure ignore que la densité de généralistes observée en France est l'une des plus élevées des pays de l'OCDE, se situant au deuxième rang après la Suisse (HCAAM, 2007). Elle masque que le vrai problème en matière d'accès aux soins n'est pas le nombre de médecins, mais leur répartition géographique.

La régulation quantitative de l'offre de médecins n'est pas une spécificité française. Des restrictions sévères ont été adoptées au Canada, pays doté d'une assurance maladie universelle et d'un système de paiement à l'acte comparables au système français. Elles ont conduit à une pénurie de médecins se traduisant par des files d'attentes (Kirby, 2002). Aux États-Unis, le contrôle du nombre de médecins a marqué l'émergence de la médecine moderne. Un marché libre des soins était en place au XIX ${ }^{\text {ème }}$ siècle, associé à des niveaux de formation médicale très hétérogènes. Les médecins américains, réunis au sein de 1'American Medical Association $(A M A)$, ont œuvré en faveur d'une réforme des études médicales et d'une homogénéisation des critères retenus pour autoriser l'exercice de la médecine. En 1910, le rapport Flexner, rédigé sous l'égide de la fondation Carnegie à la demande d'une commission de l'AMA, recommande une uniformisation de la formation et une décroissance du nombre des écoles médicales et du nombre d'étudiants admis. Des rentes de monopoles ont-elles pu résulter des restrictions à l'entrée introduites par l'AMA ? En tout cas, elles ont été appliquées avec une rigueur impressionnante : entre 1900 et 1950 , le nombre de diplômés des écoles médicales est resté constant alors que dans la même période, la population doublait et que le produit par tête était multiplié par six. En matière de revenu, la position des médecins américains par rapport aux autres professions est exceptionnelle, si on la compare à la position relative de leurs collègues dans les autres pays de l'OCDE (3). Cette élévation de la position relative des médecins aux États-Unis peut être attribuée à l'action de l'AMA en faveur de restrictions sur le nombre de médecins formés (McGuire, 2000).

Ces deux exemples montrent que la régulation de l'offre de médecins peut répondre à des objectifs très variés selon le système de soins concerné : maîtrise des dépenses dans le cadre d'un système où l'assurance est universelle et les tarifs régulés (Canada); limitation de l'offre dans un système où les tarifs sont généralement libres afin de maximiser le niveau de revenu des médecins (États-Unis). Ces exemples illustrent les enjeux associés à la régulation du nombre de médecins : le niveau de leur revenu et celui des dépenses de soins.

1. Du moins jusqu'à récemment : appliquée à partir de 2005, la loi du 13 août 2004 met en place le parcours de soins coordonnés, avec la désignation par chaque assuré d'un médecin traitant.

2. La durée des études pour être médecin généraliste est de neuf ans en 1978.

3. En moyenne, leur revenu est 4,2 fois plus élevé que le PIB par tête pour les généralistes et 6,6 fois plus élevé pour les spécialistes (OCDE, 2006). Ces valeurs les placent en tête des pays développés. Pour la France, les proportions correspondantes sont 2,8 (généralistes) et 4,6 (spécialistes). 
Comment la régulation de la démographie médicale influence-t-elle les carrières des médecins en France? En nous limitant aux généralistes du secteur 1 , nous estimons des fonctions de gains visant à expliquer leurs honoraires ou leurs revenus. Ces fonctions permettent d'identifier différents effets : celui de la date, qui mesure un choc temporel affectant tous les médecins de façon identique une année donnée ; celui de l'expérience, lié à l'évolution dans le temps de l'activité du médecin depuis son installation; celui, enfin, de la cohorte. Ce dernier effet correspond aux différences d'honoraires observables entre les générations de médecins, toutes choses égales par ailleurs, en contrôlant par les caractéristiques observables du praticien, son genre, sa localisation ou encore son avancée dans la carrière. L'estimation des effets cohorte permet d'évaluer l'impact de la démographie médicale sur les honoraires des médecins, et de comparer son ampleur à l'effet des revalorisations de tarifs conventionnels intervenues sur la période $1983-2004$.

Nous n'étudions pas seulement les honoraires des médecins, mais aussi leurs revenus : pour la première fois ces derniers sont reconstitués au niveau individuel au moyen d'une exploitation détaillée des déclarations des bénéfices non commerciaux (BNC, répertoriées dans les statistiques fiscales de la Direction Générale des Finances Publiques) et du panel de médecins de la CNAMTS.

Les résultats économétriques sont complétés par une analyse en termes de dominance stochastique. Cette approche permet de dépasser l'évaluation des différences de niveaux moyens en comparant les distributions d'honoraires entre générations.

\section{La régulation de l'offre en médecine ambulatoire en France : état des lieux}

$\mathbf{E}$ $n$ France, la régulation de l'offre en médecine ambulatoire porte sur deux volets : la régulation du nombre de médecins installés et la définition du système de paiement. Le nombre de médecins est régulé depuis 1971 par le numerus clausus. Ce dispositif a introduit une sélection sévère à la fin de la première année des études médicales : selon les années, entre $15 \%$ et $25 \%$ des étudiants sont autorisés à poursuivre leurs études (cf. encadré 1). Selon

Encadré 1

\section{LES ÉTUDES MÉDICALES}

Jusqu'en 1984, il faut sept ans d'études pour être médecin généraliste, dont un troisième cycle des études médicales (TCEM) qui dure un an. L'instauration du numerus clausus en 1971 a introduit une sélection sévère à l'issue de la première année : entre $15 \%$ et $25 \%$ des étudiants passent en deuxième année. De façon générale, le concours de première année a favorisé les redoublements, ce qui a contribué à allonger la durée des études. Une comparaison de l'organisation des études médicales en France et aux États-Unis montre que la sélection est sévère en France avec des études quasi gratuites, alors qu'aux Etats-Unis les études médicales sont coûteuses avec une sélection moins drastique: il y a en moyenne deux candidats pour un poste dans les facultés de médecine (McGuire, 2000).

Une réforme des études médicales intervenue en 1984 établit la distinction entre résidanat pour être généraliste et internat pour être spécialiste. Elle augmente la durée du TCEM à deux ans dès 1988, soit une durée des études d'au moins huit ans. La durée du résidanat sera portée à deux ans et demi en 1997 puis à trois ans en 2001. L'instauration des épreuves classantes nationales (ECN) en 2004 maintient la durée du TCEM à trois ans, soit une durée minimale d'études de neuf ans pour être médecin généraliste. Enfin, l'installation en libéral s'effectue en général une année après la thèse.

Les ECN s'imposent pour tout passage en troisième cycle, à la différence du concours de l'internat qui ne concernait que les spécialistes. Désormais, la médecine générale est une discipline comme une autre. En fonction de leur rang de classement, les futurs médecins choisissent une discipline parmi les onze proposées et un lieu de formation. En pratique, le nombre de postes offerts est supérieur au nombre de candidats moins les défections. De ce fait, les ECN n'ont pas permis de réguler la répartition des étudiants entre les disciplines.

La longueur des études médicales, jointe à l'existence d'un probable redoublement et au délai séparant la thèse de l'installation, implique que la durée séparant l'instauration d'un numerus clausus de son impact sur les effectifs médicaux peut être considérable. Les premiers médecins touchés par l'instauration du numerus clausus en 1971 ne se sont installés qu'à partir de 1980, soit neuf ans plus tard (7 ans d'études +1 redoublement +1 année avant l'installation). Par la suite, ce délai s'allonge avec l'augmentation de la durée du TCEM. 
un récent rapport du Sénat (2007), c'est pour contenir la progression des dépenses de santé que le numerus clausus a été mis en place.

\section{Les fluctuations du numerus clausus}

Initialement fixé à 8588 places, le numerus clausus est resté stable à ce niveau relativement élevé pendant plusieurs années. Ce n'est qu'à partir de la fin des années 1970 que les syndicats de médecins prennent massivement conscience qu'un nombre excessif de praticiens peut constituer une menace pour leurs revenus (Déplaude, 2007). Leur mobilisation conduit à une réduction du numerus clausus. Ainsi, une véritable politique de restriction n'est effective qu'à partir de 1978. Compte tenu de la longueur des études médicales (cf. encadré 1), son effet ne peut être observé qu'à partir des années 1987 et 1988.

Les restrictions sur le numerus clausus sont restées sévères jusqu'en 1993, année où le niveau le plus bas est atteint, avec 3500 places. Un dispositif transitoire d'incitation à la retraite anticipée (4) est instauré entre 1988 et 2003 pour renforcer cette politique de réduction du nombre de médecins en activité. Le départ prévisible des générations nombreuses de médecins issues du baby-boom conduit à un renversement de perspectives à partir des années 2000. Le discours dominant ne dénonce plus la pléthore mais la pénurie de médecins. Le gouvernement redresse alors progressivement le numerus clausus : avec 7300 places en 2008, celui-ci se rapproche de son niveau initial.

Concernant la localisation des médecins, peu de mesures ont été prises jusqu'à une période très récente. Les dernières variations du numerus clausus auraient pu être utilisées à des fins de répartition géographique. Or, il semble qu'elles aient été ventilées par université avec un taux directeur unique (Cour des Comptes, 2007). Depuis 2004 ont été instaurées de nombreuses incitations financières à l'installation dans des zones sous-médicalisées : une majoration de $20 \%$ de la rémunération des généralistes, des dispositifs d'exonération fiscale dont la valeur moyenne s'élève à 37000 euros par bénéficiaire, auxquels s'ajoutent de très nombreuses aides locales ou régionales. Compte tenu de la date récente de leur instauration, les effets de ces aides n'ont pas encore pu être évalués, mais ils ne sont pas manifestes.

\section{La plupart des généralistes libéraux appartiennent au secteur 1 , où s'appliquent les tarifs conventionnels}

Pour les soins ambulatoires, l'exercice libéral de la médecine avec un paiement à l'acte et des tarifs fixés dans le secteur 1 domine très largement le paysage national. Certains médecins libéraux peuvent percevoir des compléments salariaux, mais ils sont minoritaires, surtout chez les omnipraticiens : parmi les médecins ayant une activité libérale, 77,1\% sont exclusivement libéraux ; chez les omnipraticiens cette proportion atteint 90,1 \% (HCAAM, 2007). Deux secteurs sont définis pour la tarification des actes : dans le secteur 1, les tarifs sont fixés par des conventions nationales et servent de référence pour les remboursements effectués par la sécurité sociale et les contrats d'assurances complémentaires. Un secteur 2 a été ouvert en 1980 pour autoriser des dépassements. Ces derniers ne sont pas pris en charge par la sécurité sociale, mais par certaines assurances complémentaires ( $40 \%$ des assurés sociaux n'ont toutefois pas de couverture pour les dépassements). L'accès au secteur 2 a été fermé en 1990, sauf pour les médecins qui s'installent pour la première fois après une expérience professionnelle de chef de clinique ou d'assistant des hôpitaux. En pratique, la question des dépassements se pose peu pour les omnipraticiens, dont $13 \%$ seulement appartiennent au secteur 2 en 2005 (Eco-santé, 2007). Elle est en revanche particulièrement aiguë pour les spécialistes, qui sont très nombreux en secteur 2 (près de la moitié) avec un taux de dépassement moyen de $51 \%$ en 2005 (Cour des Comptes, 2007).

Le champ de notre étude concerne les médecins généralistes, devenus le pivot de l'organisation de la médecine ambulatoire depuis le dispositif du médecin traitant introduit en 2004. Comme ils sont très majoritairement en secteur 1 où les tarifs sont fixés, le système de paiement à l'acte se traduit, pour ces médecins, par une liaison étroite entre leurs honoraires et le nombre d'actes qu'ils ont délivrés.

\section{Les enjeux de la régulation de l'offre médicale}

Un rapide état des lieux de la médecine ambulatoire en France ne permet pas de repérer de dis-

4. Le MICA (mécanisme d'incitation à la cessation d'activité), a rencontré un grand succès auprès des médecins libéraux, avant d'être supprimé et remplacé par des actions à la finalité inverse, visant à prolonger l'activité par des possibilités de cumul emploi retraite (Cour des Comptes, 2007). 
fonctionnements majeurs, mais des problèmes récurrents dont les conséquences pourraient s'aggraver à terme.

Comme nous l'avons mentionné en préambule, la France est un des pays de l'OCDE où la densité de généralistes est la plus élevée. Cependant, une mauvaise répartition géographique des médecins entraîne des inégalités dans l'accès aux soins et la possibilité d'un rationnement des patients au niveau local (5).

L'introduction des épreuves classantes nationales (cf. encadré 1) a révélé la faible attractivité de la médecine générale : $14 \%$ des postes offerts en médecine générale n'ont pas été pourvus en 2006 ; cette proportion atteignait $40 \%$ en 2005 (Billaut, 2006 ; Vanderschelden, 2007). On observe aussi une diminution de la proportion d'étudiants en médecine désireux de s'installer en libéral (Bourgueil, 2007).

Enfin, des comportements de demande induite ont été identifiés pour les médecins généralistes du secteur 1 (Delattre et Dormont, 2003, 2005). Lorsque la densité dans leur zone d'exercice augmente, les médecins subissent un rationnement de leur nombre de consultations car ils sont plus nombreux à se partager le même nombre de patients. Des estimations ont montré que ces rationnements sont en partie compensés par une augmentation du volume de soins délivrés au cours de chaque consultation. Ces comportements sont plus marqués dans les départements où la densité est élevée.

La régulation de l'offre en médecine ambulatoire doit garantir à tous les citoyens l'accès à des soins répondant à leurs besoins et une égalité dans cet accès. Cet objectif nécessite des médecins en nombre suffisant. Dans ce but, il convient de maintenir l'attractivité de la profession médicale pour les futurs étudiants, en particulier celle de la médecine générale en libéral. Cette attractivité dépend des revenus des médecins, mais aussi des écarts potentiels entre générations.

Ces principes sont énoncés dans l'hypothèse où le système de paiement à l'acte ne serait pas remis en cause dans un futur proche - une hypothèse raisonnable dans le contexte français.

\section{Une évolution très heurtée de la démographie médicale ...}

La population étudiée est celle des omnipraticiens libéraux du secteur 1, pour laquelle nous disposons d'un échantillon représentatif sur les années 1983 à 2004 pour les cohortes 1945 à 2003 (la cohorte est définie par l'année d'installation en libéral) (cf. encadré 2). Sur le modèle de la pyramide des âges, nous avons construit la « pyramide des cohortes » des médecins (cf. graphique I). L'allure de cette pyramide ne correspond pas du tout à l'idéal théorique qui résulterait de l'application d'un niveau de densité médicale optimal (et constant) à une population en croissance. Afin de permettre l'interprétation de sa forme accidentée, nous avons représenté, avec cette pyramide, le nombre de naissances observées trente ans avant la date d'installation (l'âge moyen d'installation est de trente ans) (cf. graphique I) et la valeur du numerus clausus 9 et 10 ans auparavant (cf. graphique II).

L'augmentation très importante des effectifs des cohortes des années 1974-1978 s'explique par l'arrivée des générations nombreuses du babyboom. En l'absence de numerus clausus, celle-ci se traduit par un saut dans les effectifs des étudiants en médecine. Instauré à la rentrée 1971, le numerus clausus introduit une déconnection entre la courbe plutôt croissante décrivant le nombre des naissances trente ans auparavant et le profil déclinant des effectifs des médecins nouvellement installés (partie droite du graphique I). L'impact du numerus clausus apparaît clairement sur le graphique II. Après avoir été constant pendant plusieurs années, le numerus clausus décroît à partir de la fin des années 1970 . Dans le même temps, la durée des études médicales augmente d'une année, passant de 9 ans à 10 ans : ce n'est qu'à partir de la fin des années 1980 que les effectifs des nouveaux médecins décroissent fortement et continûment.

Le faible nombre de médecins figurant dans les cohortes antérieures à 1970 est dû aux départs à la retraite. Ceux-ci résultent de l'âge des personnes concernées mais aussi du mécanisme d'incitation à la cessation d'activité (Mica), mis en place entre 1988 et 2003 pour encourager la retraite anticipée : un quart des médecins âgés de 57 à 65 ans ont bénéficié de ce dispositif.

\section{...qui se traduit par des différences d'honoraires entre cohortes}

Les honoraires moyens par cohorte et expérience sont représentés dans le graphique III.

5. Le risque de pénuries locales semble limité pour le moment: une analyse au niveau cantonal évalue que la proportion de la population concernée par des difficultés d'accès aux soins se situerait dans une fourchette allant de $0,6 \%$ à $4,1 \%$ (ONDPS, 2005). 
On obtient une allure générale en U renversé, caractéristique des effets de l'expérience. Mais ce graphique est difficilement interprétable : les cohortes étant observées en différents points du temps, leurs positionnements respectifs sont affectés par les revalorisations tarifaires.

Une approche plus pertinente, suggérée par Koubi (2003a) consiste à représenter les honoraires moyens par cohorte et expérience, nets de l'effet de date (cf. graphique IV). Afin d'améliorer la lisibilité du graphique, nous n'avons considéré que les cohortes correspondant aux années 1965, 1972, 1977, 1985, 1993 et 1999, lesquelles sont signalées explicitement sur la pyramide des cohortes (cf. graphique I). À expérience donnée, la situation financière des médecins atteint son apogée pour la cohorte 1972. Elle se dégrade ensuite pour la cohorte 1977, pour la cohorte 1985 et plus encore pour la cohorte 1993.

On observe donc des différences d'honoraires entre les générations de médecins. Ces diffé- rences semblent toutefois d'une ampleur plus limitée que les écarts dus à la variation de l'expérience. L'analyse économétrique qui suit va permettre d'estimer plus précisément les effets de l'expérience, de la cohorte et de la date, afin d'évaluer leur importance respective.

\section{Analyse économétrique des carrières des médecins généralistes}

Vous estimons une fonction de gains. 1 Introduite par Mincer (1974), cette spécification est couramment utilisée pour l'analyse des carrières salariales. Son contenu théorique est toutefois différent lorsqu'il s'agit d'étudier des salaires ou des rémunérations de médecins. Dans l'analyse des salaires, on cherche à évaluer le rendement du capital humain initial et l'effet de l'expérience est interprété comme celui du capital humain accumulé au cours de l'activité

Graphique I

Pyramide des cohortes et nombre de naissances 30 ans auparavant

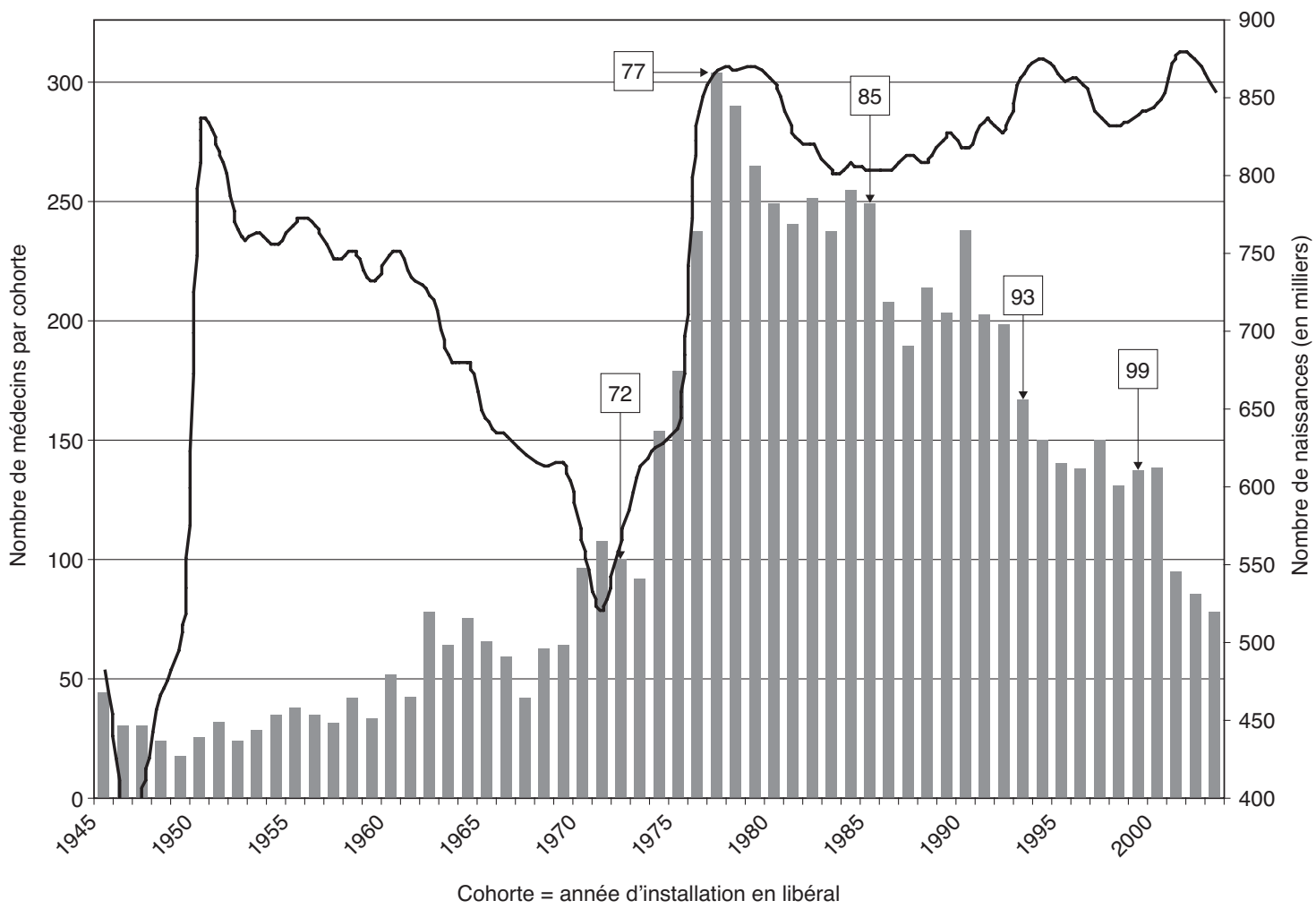

Nombre de médecins _ Nombre de naissances 30 ans avant

Lecture : on entend par cohorte l'ensemble des médecins qui se sont installés une année donnée et qui sont observés dans l'échantillon (période 1983-2004). Cinq cohortes (1972, 1977, 1985, 1993, 1999) sont distinguées au moyen d'étiquettes : il s'agit de cohortes analysées en détail tout au long de l'étude.

Champ : médecins installés entre 1945 et 2004 présents au moins une fois dans la base entre 1983 et 2004 (soit 7216 au total). Source : panel d'omnipraticiens libéraux du Système National Inter Régimes (SNIR,CNAMTS), 1983-2004. 
professionnelle. Les médecins de notre échantillon sont homogènes du point de vue de leur capital humain initial. L'effet de l'expérience sur leurs honoraires dépend de la dynamique de constitution de leur clientèle plutôt que de leur expérience accumulée. Pour un salarié, l'effet positif de l'expérience sur le salaire découle de l'amélioration de sa productivité horaire. $A$ contrario, la rémunération de la consultation est la même pour tous les médecins du secteur 1 (où les tarifs sont fixes), quelle que soit leur expérience. Une éventuelle amélioration de la productivité peut se traduire, pour le médecin, par un raccourcissement de la durée de la consultation lui permettant de recevoir plus de patients par jour et d'améliorer ainsi son revenu. On peut aussi concevoir que la qualité des soins prodiguée par le médecin s'améliore avec l'expérience. Mais ce deuxième effet n'a pas d'impact sur le revenu si le nombre des consultations n'est pas modifié.
Une autre différence importante existe entre les carrières des médecins et celles des salariés : les médecins ont plus de marges de manœuvre dans la détermination de leur durée de travail que les salariés. Ces derniers sont souvent contraints sur leur durée du travail, avec un choix plutôt restreint entre temps plein ou temps partiel, lorsqu'un choix existe.

\section{Les facteurs explicatifs de l'activité et des honoraires des médecins généralistes}

L'approche économétrique est détaillée dans l'encadré 3. La spécification retenue explique le logarithme des honoraires (6) $y_{i c t}$ du médecin $i$ appartenant à la cohorte $c$ et observé en $t$ par la densité médicale des omnipraticiens et la den-

6. Dans tout l'article, les honoraires et les revenus (estimés) sont déflatés par l'indice des prix à la consommation.

\section{Graphique II}

Pyramide des cohortes et valeur du numerus clausus 9 ou 10 ans avant la date d'installation

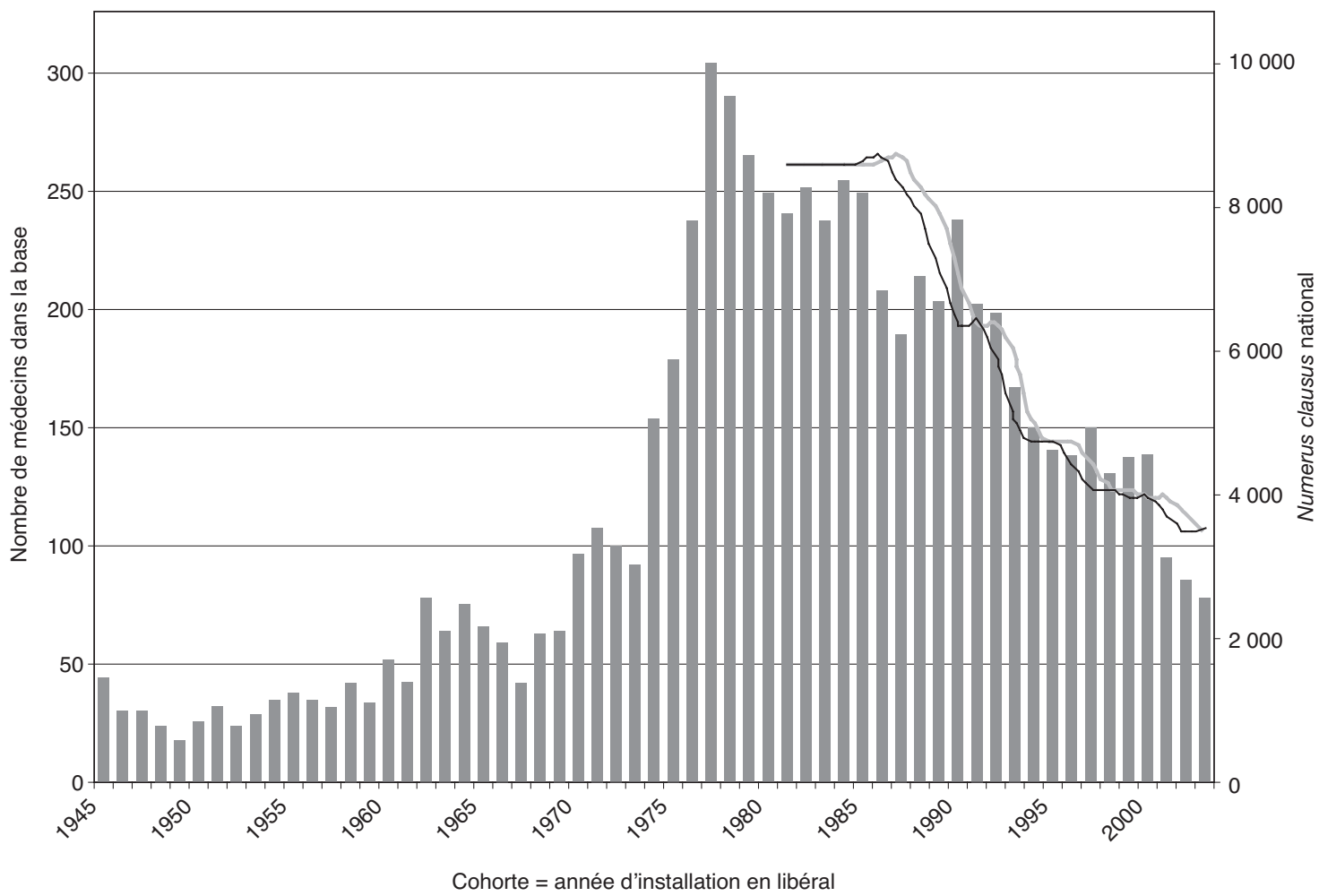

Cohorte = année d'installation en libéral

\footnotetext{
Nombre de médecins

NC 9 ans avant

NC 10 ans avant

Lecture : le nombre de médecins installés entre 1980 et 1988-1989 est influencé par la valeur du numerus clausus neuf ans auparavant (cf. encadré 1). Pour les médecins installés entre 1989 et 2000, les études de médecine ont duré un an de plus ; leurs effectifs sont à comparer au numerus clausus 10 ans auparavant. La durée des études des médecins installés entre 2001 et 2004 puis après 2005 est augmentée d'un semestre puis d'une année; les effectifs de ces cohortes seraient comparables à la valeur du numerus clausus 11 ans auparavant (non représentée sur le graphique).

Champ : médecins du secteur 1, installés entre 1945 et 2004 et présents au moins une fois dans la base entre 1983 et 2004 (soit 7216 au total).

Source : Panel d'omnipraticiens libéraux du SNIR (CNAMTS), 1983-2004.
} 
sité médicale des spécialistes dans son département d'exercice, par des variables décrivant le médecin et son type d'activité et des variables indicatrices de la région d'installation.

La spécification estimée vise à décrire le niveau d'honoraires du médecin déterminé par l'équilibre entre l'offre et la demande de soins sur le marché de la médecine ambulatoire. Il est traditionnel de supposer que les médecins sont en concurrence monopolistique : leurs services ne sont pas parfaitement substituables aux yeux des patients à cause de différences dans leur localisation (distance pour accéder au médecin) et dans la qualité des soins. Chaque médecin maximise son utilité pour déterminer son offre de soins, sous la double contrainte de la demande qui s'adresse à lui et de la fonction de production de soins. La production de soins dépend positivement du temps de travail et négativement de la durée de la consultation (en France, sur la période étudiée, les tarifs ne sont pas différenciés en fonction de cette durée). La concurrence est plus intense lorsque le nombre de médecins augmente : la demande qui s'adresse à chaque médecin dépend négativement du nombre de

\section{Graphique IV \\ Honoraires moyens nets de l'effet de date, par cohorte et expérience}

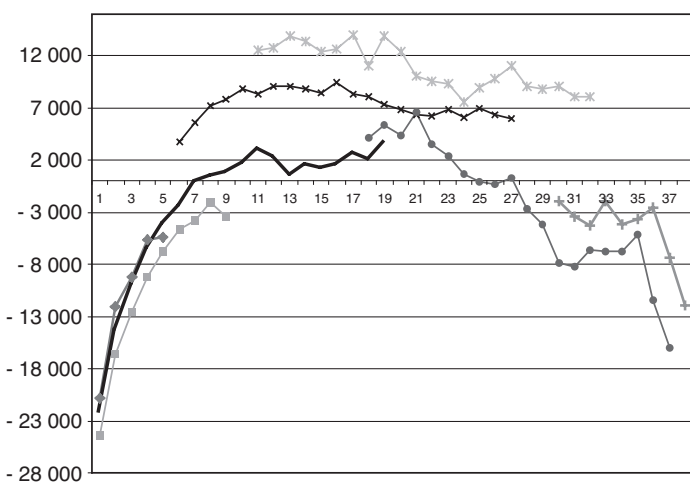

\begin{tabular}{|llll|}
\hline$\rightarrow 1999$ & -1993 & -1985 \\
$\rightarrow 1972$ & $\rightarrow 1965$ & +1953 & \\
\hline - 1972 &
\end{tabular}

Lecture : soit hono ${ }_{i c t}$ les honoraires du médecin i appartenant à la cohorte $c$ et observé à l'année t. On a représenté les valeurs de hono - hono , où hono désigne la moyenne des honoraires par cohorte êt année et hono la moyenne des honoraires par année. Ces honoraires sont exprimés en euros constants de 2004. Pour améliorer la lisibilité du graphique, seules sept cohortes sont représentées. II s'agit des cohortes distinguées précédemment dans la pyramide des cohortes (graphique I).

Champ : médecins du secteur 1, installés entre 1945 et 2004 et présents au moins une fois dans la base entre 1983 et 2004 (soit 7216 au total).

Source: panel d'omnipraticiens libéraux du SNIR (CNAMTS), 1983-2004.

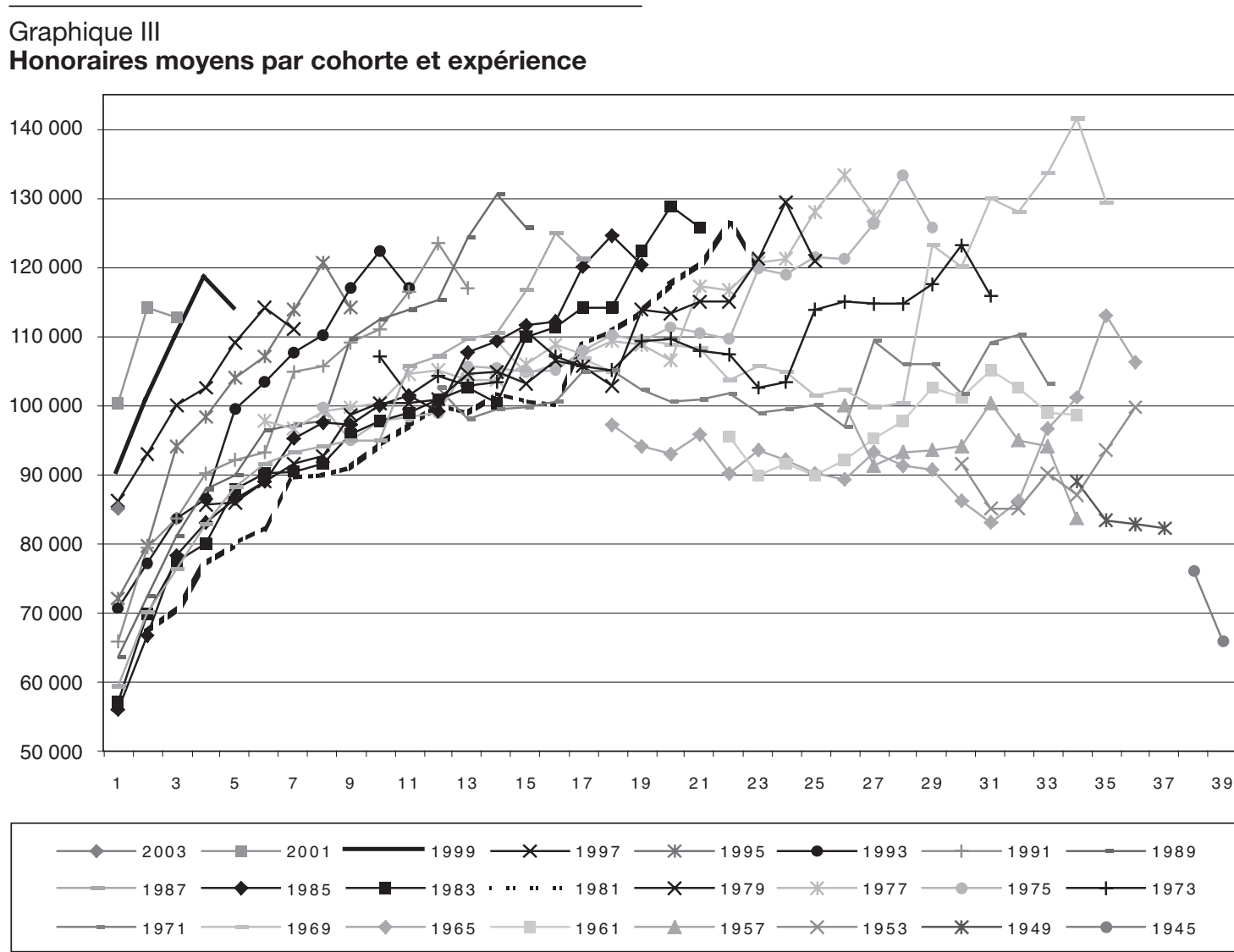

Lecture : les honoraires moyens annuels sont exprimés en euros constants de 2004, déflatés par l'indice des prix à la consommation.. Champ : médecins du secteur 1, installés entre 1945 et 2004 et présents au moins une fois dans la base entre 1983 et 2004 (soit 7216 au total).

Source : panel d'omnipraticiens libéraux du SNIR (CNAMTS), 1983-2004. 
Encadré 2

\section{UN PANEL REPRÉSENTATIF DES OMNIPRATICIENS LIBÉRAUX FRANÇAIS}

Cette étude exploite les données d'un panel représentatif des omnipraticiens libéraux français. Le terme omnipraticiens englobe les généralistes et les médecins possédant un "mode d'exercice particulier » (MEP) comme l'acupuncture ou l'homéopathie. Après apurement de la base de données d'origine et sélection des observations correspondant au champ retenu, l'échantillon comporte 91634 observations relatives à 7216 médecins sur la période 1983-2004. Tous les médecins ne sont pas observés sur toute la période : l'échantillon est non cylindré car représentatif des entrées et sorties d'activité intervenues chaque année.

\section{Base de données originelle}

Le panel d'omnipraticiens libéraux fourni par la CNAMTS reprend les statistiques relevées en date de remboursement par le Système national inter régimes (SNIR). Sont renseignés le montant des honoraires des omnipraticiens (honoraires annuels, dépassements présentés au remboursement et frais de déplacement), la mesure de leur activité annuelle et sa décomposition en consultations, visites et actes cotés), l'année de thèse et la date d'installation en libéral, la région et le département d'exercice, le secteur conventionnel, le mode d'exercice (libéral à temps plein ou à temps partiel), la présence ou non d'un mode d'exercice particulier (acupuncteur, homéopathe, etc.), ainsi que des caractéristiques démographiques comme le sexe et l'année de naissance.

Obtenu par tirage dans les données administratives exhaustives de tous les médecins nés au mois de mai, ce panel est représentatif de la population des omnipraticiens en exercice sur les années 1979-2004. Les médecins partant à la retraite sortent chaque année de l'échantillon, lequel est complété par un tirage dans la population des nouveaux installés.

\section{Champ de l'étude}

Les années d'installation ou de cessation d'activité sont des années d'activité incomplètes : les observations correspondantes sont éliminées de l'échantillon de travail. Du champ de l'étude sont aussi exclus les médecins non conventionnés et les praticiens hospitaliers à temps plein. Sont donc considérés les médecins libéraux à temps plein ou à temps partiel, ces derniers étant distingués dans les estimations économétriques par une variable indicatrice. Pour des raisons de fiabilité de l'information, les omnipraticiens exerçant dans les Dom sont également exclus et seules les années 1983 à 2004 sont conservées.

On conserve toutes les cohortes 1945 à 2003 pour l'analyse descriptive en termes de pyramide de cohortes. En revanche, il convient de réduire le champ d'investigation aux cohortes 1970 à 2001 pour l'approche économétrique. En effet, les cohortes anciennes (1945 à 1969) et très récentes (2002-2003) comprennent trop peu de médecins (entre 12 et 85 ) pour per- mettre une inférence statistique robuste. Un échantillon de 6016 médecins est donc utilisé pour l'analyse économétrique (avec 81691 observations).

Enfin, nous limitons le champ de l'étude aux médecins du secteur 1. En dehors du fait que leurs comportements obéissent vraisemblablement à des logiques économiques très différentes de celles de leurs collègues du secteur 1 , les médecins du secteur 2 présentent de fortes hétérogénéités non observées qui rendent nécessaire un traitement séparé.

\section{Richesse de l'information disponible}

Le tableau A résume la structure de l'échantillon. II donne une idée de la richesse de l'information disponible : 32 cohortes comprenant chacune de 95 à 290 médecins sont observées sur la période 1983-2004. Cet échantillon est exceptionnel à cause de la longueur de la période et du nombre de cohortes observées. II est particulièrement intéressant et presque unique en son genre, car il fournit une information fiable sur les honoraires d'une profession libérale. C'est une conséquence de la spécificité du système de santé français, où les consultations chez les médecins libéraux sont remboursées par un assureur unique (c'est-à-dire où les honoraires de cette profession libérale sont financés par des prélèvements obligatoires).

\section{Construction des revenus des omnipraticiens à partir de données fiscales}

Évaluer les revenus des médecins est crucial dès qu'il s'agit de raisonner en termes de niveau de vie ou d'attractivité de la profession médicale par rapport à d'autres professions de niveau de formation comparable. Répertoriées dans les statistiques fiscales de la Direction Générale des Finances Publiques et disponibles pour les années 1993 à 2004, les déclarations des BNC (bénéfices non commerciaux) nous ont permis d'estimer les revenus individuels. Grâce à ce travail original, notre base de données représente, à ce jour, la seule source disponible sur longue période comportant en plus des variables initiales les revenus et les charges individuels.

Notre méthode se distingue de celle utilisée jusqu'à présent par la Drees, laquelle publie régulièrement des informations sur le niveau et l'évolution des revenus libéraux des omnipraticiens. Ces derniers sont calculés en appliquant aux honoraires issus du SNIR le taux de débours et rétrocessions moyen et le taux de charge moyen observés dans les BNC (Legendre, 2007). Cette méthode repose sur des indicateurs moyens qui gomment les disparités individuelles. Or, la dispersion des revenus est affectée à la fois par la dispersion des honoraires et la dispersion des taux de charges. Ces derniers sont très variables d'un omnipraticien à l'autre. Ils dépendent de la commune d'installation, de la région d'exercice, de l'organisation ou non en cabinet de groupe et de l'ancienneté (BreuilGenier, 2003). De façon générale, une forte proportion, 
Encadré 2 (suite)

de charges fixes fait dépendre le taux de charge du niveau d'activité et d'honoraires. Les données issues des BNC fournissent en 2003 une valeur médiane du taux de charges de $44 \%$; ce taux varie de $39 \%$ (premier décile) à 55\% (neuvième décile).

Au niveau individuel, on a :

Revenu = honoraires bruts - débours et rétrocessions charges

Les débours et rétrocessions sont les honoraires reversés par un médecin à son remplaçant, les chèques impayés et les actes non payés. Ils sont observés dans les BNC, tout commeles charges individuelles. Notre travail consiste à jouer sur les deux bases de données : le panel d'omnipraticiens et les données BNC. Nous spécifions des équations visant à expliquer le taux de débours et rétrocessions d'une part et les charges d'autre part. L'estimation de ces modèles sur les données BNC permet de construire des prédictions au niveau individuel, qui sont ensuite appliquées aux honoraires bruts individuels observés dans le panel.

(i). Le taux de débours et rétrocessions représente en moyenne $3,5 \%$ des honoraires bruts. II est estimé

\section{Tableau A}

\section{Description de l'échantillon initial}

\begin{tabular}{|c|c|c|c|c|}
\hline $\begin{array}{l}\text { Cohorte d'installation } \\
\text { en libéral }\end{array}$ & Nombre d'observations & Nombre de médecins & Période d'observation & $\begin{array}{l}\text { Valeurs observées pour } \\
\text { l'expérience (1) }\end{array}$ \\
\hline 1970 & 1290 & 97 & $1983-2004$ & $13-34$ \\
\hline 1971 & 1565 & 107 & $1983-2004$ & $12-33$ \\
\hline 1972 & 1656 & 100 & 1983-2004 & $11-32$ \\
\hline 1973 & 1549 & 92 & $1983-2004$ & $10-31$ \\
\hline 1974 & 2539 & 154 & $1983-2004$ & $9-30$ \\
\hline 1975 & 3014 & 179 & $1983-2004$ & $8-29$ \\
\hline 1976 & 3961 & 238 & $1983-2004$ & $7-28$ \\
\hline 1977 & 5154 & 304 & $1983-2004$ & $6-27$ \\
\hline 1978 & 5129 & 290 & $1983-2004$ & $5-26$ \\
\hline 1979 & 4609 & 265 & $1983-2004$ & $4-25$ \\
\hline 1980 & 4011 & 250 & $1983-2004$ & $3-24$ \\
\hline 1981 & 4256 & 241 & $1983-2004$ & $2-23$ \\
\hline 1982 & 4107 & 252 & $1983-2004$ & $1-22$ \\
\hline 1983 & 3837 & 237 & $1984-2004$ & $1-21$ \\
\hline 1984 & 4095 & 255 & $1985-2004$ & $1-20$ \\
\hline 1985 & 3881 & 250 & 1986-2004 & $1-19$ \\
\hline 1986 & 3276 & 208 & $1987-2004$ & $1-18$ \\
\hline 1987 & 2764 & 190 & 1988-2004 & $1-17$ \\
\hline 1988 & 2972 & 215 & 1989-2004 & $1-16$ \\
\hline 1989 & 2658 & 204 & $1990-2004$ & $1-15$ \\
\hline 1990 & 2929 & 238 & $1991-2004$ & $1-14$ \\
\hline 1991 & 2306 & 202 & $1992-2004$ & $1-13$ \\
\hline 1992 & 2183 & 201 & 1993-2004 & $1-12$ \\
\hline 1993 & 1561 & 167 & 1994-2004 & $1-11$ \\
\hline 1994 & 1246 & 149 & $1995-2004$ & $1-10$ \\
\hline 1995 & 1113 & 150 & 1996-2004 & $1-9$ \\
\hline 1996 & 1001 & 139 & $1997-2004$ & $1-8$ \\
\hline 1997 & 906 & 151 & $1998-2004$ & $1-7$ \\
\hline 1998 & 730 & 131 & 1999-2004 & $1-6$ \\
\hline 1999 & 620 & 137 & $2000-2004$ & $1-5$ \\
\hline 2000 & 509 & 138 & 2001-2004 & $1-4$ \\
\hline 2001 & 264 & 95 & $2002-2004$ & $1-3$ \\
\hline $\begin{array}{l}1945-1969 \\
2002-2003\end{array}$ & \multicolumn{4}{|c|}{$\begin{array}{l}\text { Il y a entre } 12 \text { et } 85 \text { médecins par cohorte, ce qui n'est pas suffisant pour mener une analyse écono- } \\
\text { métrique robuste. }\end{array}$} \\
\hline Total (2) & 81691 & 6016 & $1983-2004$ & $1-34$ \\
\hline
\end{tabular}

1. L'expérience est définie comme l'année d'observation moins l'année de début d'activité, moins la durée de l'interruption de carrière, s'il y a lieu.

2. Ces chiffres sont calculés à partir de l'échantillon utilisé pour l'analyse économétrique : seules les cohortes 1970 à 2001 sont conservées.

Champ : médecins installés entre 1945 et 2004 présents au moins une fois dans la base entre 1983 et 2004 (soit 7216 au total). Source : panel d'omnipraticiens libéraux du SNIR (CNAMTS), 1983-2004. 
médecins exerçant dans le même département et positivement de la durée de la consultation, laquelle est supposée améliorer la qualité des soins. Comme les tarifs sont fixés et identiques dans le secteur 1, l'équilibre entre l'offre et la demande est obtenu dans cette formalisation par les ajustements sur la durée de la consultation.

Dans le secteur 1, les honoraires du médecin dépendent seulement de la quantité de soins fournis puisque les tarifs sont fixés. Notre représentation du marché des soins ambulatoires permet de comprendre que cette quantité de soins dépend, du côté de l'offre, des préférences du médecin concernant la consommation et le loisir, et, du côté de la demande, du nombre de médecins concurrents dans le même département.

La spécification retenue s'écarte de l'approche classique en ne formalisant pas l'effet de l'expérience par une fonction polynomiale. Afin d'exploiter au mieux la richesse de l'information offerte par l'échantillon, nous avons choisi de conserver une forme très souple en spécifiant par des constantes spécifiques les effets de

Encadré 2 (fin)

à l'aide d'un modèle Tobit généralisé afin de teni compte du fait que la moitié des observations environ sont égales à zéro. L'équation de sélection utilise comme variables explicatives les honoraires bruts et la densité d'omnipraticiens dans le département d'exercice. Le modèle de régression spécifie le taux des débours et rétrocessions par une fonction quadratique des honoraires bruts. L'estimation obtenue est alors appliquée aux honoraires bruts du panel pour calculer les honoraires nets.

(ii). Les charges individuelles sont estimées à partir des honoraires nets, seule variable explicative commune aux deux bases de données. Quatre stratégies de spécification et/ou d'estimation sont considérées: régression médiane, estimation par moindres carrés ordinaires d'une fonction linéaire par morceaux des honoraires nets ou d'un polynôme d'ordre 2 ou 3 des honoraires nets. Les résultats sont très peu sensibles au choix de la méthode, mais nous avons conservé les estimations issues de

ces différentes stratégies afin de pouvoir vérifier la robustesse des résultats obtenus ultérieurement sur les revenus par rapport au choix retenu pour leur construction.

Dans le tableau B, le revenu est construit en utilisant un polynôme d'ordre 3 des honoraires nets pour estimer le montant des charges. Ce revenu construit sera appelé par la suite « revenu estimé ». La valeur moyenne du revenu publié par la Drees s'élève en 2004 à $61805 €$. Nous obtenons $61360 €$. Les dispersions sont plus marquées quand on considère les revenus plutôt que les honoraires (cf. tableau B). Cette différence apparaît principalement sur l'indicateur D9/D1, reflétant la plus grande variabilité des taux de charge en bas de la distribution des honoraires. Par ailleurs, on retrouve sur les revenus le resserrement des dispersions observé sur les honoraires par Samson (2006) : comme pour les honoraires, il est dû à une plus forte croissance des revenus du bas de la distribution, situés en dessous du premier décile, voire du premier quartile.

\section{Tableau B}

Évolution de la distribution des honoraires et des revenus estimés (en euros constants de 2004)

\begin{tabular}{|c|c|c|c|c|c|c|}
\hline & \multicolumn{3}{|c|}{ Honoraires } & \multicolumn{3}{|c|}{ Revenus } \\
\hline & 1983 & 2004 & $\begin{array}{c}\text { Évolution 1983- } \\
2004 \text { (en \%) }\end{array}$ & 1993 & 2004 & $\begin{array}{c}\text { Évolution 1993- } \\
2004 \text { (en \%) }\end{array}$ \\
\hline $1^{\text {er }}$ décile & 37318 & 59434 & +59 & 18162 & 25661 & +42 \\
\hline $1^{\text {er }}$ quartile & 58532 & 84344 & +44 & 31995 & 40483 & +26 \\
\hline Médiane & 88976 & 114023 & +28 & 49206 & 58555 & +19 \\
\hline Moyenne & 92165 & 118663 & +39 & 51248 & 61360 & +19 \\
\hline $3^{\text {ème }}$ quartile & 120468 & 149443 & +24 & 67844 & 79547 & +17 \\
\hline $9^{\text {ème }}$ décile & 149053 & 183482 & +23 & 86937 & 100555 & +16 \\
\hline Q3/Q1 & 2,06 & 1,77 & $-0,29$ & 2,12 & 1,96 & $-0,16$ \\
\hline D9/D1 & 3,99 & 3,08 & $-0,91$ & 4,78 & 3,92 & $-0,86$ \\
\hline D5/D1 & 2,38 & 1,91 & $-0,47$ & 2,71 & 2,28 & $-0,43$ \\
\hline D9/D5 & 1,67 & 1,61 & $-0,06$ & 1,77 & 1,71 & $-0,06$ \\
\hline
\end{tabular}

Lecture : les évolutions concernent la période 1983-2004 pour les honoraires et la période 1993-2004 pour les revenus estimés. Ces évolutions sont calculées à partir des honoraires et revenus mesurés en euros constants de 2004. Nous considérons des variations

relatives dans la partie haute du tableau (niveaux) et des variations absolues dans la partie basse (dispersions).

Champ : médecins installés entre 1945 et 2004 présents au moins une fois dans la base entre 1983 et 2004

Source : panel d'omnipraticiens libéraux du SNIR (CNAMTS), 1983-2004. 
Encadré 3

\section{SPÉCIFICATION ET ESTIMATION D'UNE FONCTION DE GAINS POUR LES MÉDECINS}

Soit $y_{\text {ict }}$ le logarithme des honoraires (ou de l'activité ou des revenus) du médecin $i$ appartenant à la cohorte $c$ et observé en $t$. Les estimations sont réalisées pour les cohortes 1970 à 2001, observées sur les années 1983 à 2004, avec des niveaux d'expérience allant de 1 à 34 ans. On considère la spécification suivante :

$y_{i c t}=a+D^{\prime}{ }_{i c t} b+Z^{\prime}{ }_{i c} d+\eta_{r}+\alpha_{e}+\delta_{t}+\gamma_{c}+\varepsilon_{i c t}$

avec

$i=1, \ldots . N ; C=1 \ldots . . C ; t=1 \ldots . T ; e=1 \ldots . E$ et

$r=1, \ldots R$

où :

$D_{\text {ict }}^{\prime}$ représente les variables explicatives qui varient dans le temps: densité médicale des omnipraticiens et densité des spécialistes dans le département d'exercice du médecin $i$;

$Z_{i c}^{\prime}$ les variables explicatives propres au médecin $i$ appartenant à la cohorte $c$ et constantes dans la dimension temporelle: sexe, durée entre année de thèse et année d'installation en libéral, type d'activité en libéral (à temps complet ou non), présence ou non d'un mode d'exercice particulier, type d'aire urbaine ;

$\alpha_{e}, e=1, \ldots, 34$ l'effet fixe (paramètre) spécifique à l'expérience, définie par le nombre d'années écoulées depuis l'installation en libéral (moins les années d'interruption de carrière, s'il y a lieu) ;

$\delta_{t}, t=1983 \ldots .2004$ l'effet fixe (paramètre) spécifique à l'année $t$;

$\gamma_{c}, c=1970, \ldots, 2001$ l'effet fixe (paramètre) spécifique à la cohorte, définie par l'année de l'installation en libéral :

$\eta_{r}$ un effet spécifique à la région d'exercice du médecin ( $r$ = lle-de-France, Centre, Nord, Picardie, etc.)

Contrairement à l'approche standard, l'effet de l'expérience n'est pas spécifié par une forme polynomiale. On choisit de conserver une grande flexibilité en utilisant des effets fixes pour spécifier les effets temporels, d'expérience et de cohorte. Une telle spécification n'est pas identifiable sans l'ajout de contraintes sur les effets. Nous avons adopté les contraintes suivantes :

$$
\begin{aligned}
& \sum_{r} \eta_{r}=0, \sum_{e} \alpha_{e}=0, \sum_{t} \delta_{t}=0 \text { et } \sum_{c} \gamma_{c}=0 \\
& \sum_{c} c * \gamma_{c}=0
\end{aligned}
$$

La contrainte (2) est sans contenu. Elle revient à définir une modalité de référence pour chacun des quatre effets, lesquels sont alors interprétables comme des contrastes à la constante.

La contrainte (3) est liée à l'existence d'une colinéarité entre les variables de date, de cohorte et d'expérience.
Pour un médecin $i$, on a en général $t=c+e$. Par exemple, en 1985, les médecins de la cohorte 1970 ont 15 ans d'expérience. Ceci n'est pas vrai pour tous les médecins de l'échantillon, car certains connaissent des interruptions de carrière. Pour ces derniers, l'expérience est calculée comme la différence entre l'année d'observation $t$ et l'année d'installation $c$, moins la durée de l'interruption. Ce cas de figure concerne $6 \%$ des observations : $t$ reste fortement corrélé avec $c+e$.

La contrainte (3) permet l'identification du modèle en imposant une absence de tendance sur l'effet cohorte. Lollivier et Payen (1990) adoptent cette contrainte pour étudier les salaires dans le cadre d'un modèle de carrière avec des effets fixes. En revanche, Audric (2006) retient une autre contrainte identifiante en imposant une absence de tendance sur les effets temporels. Dans le cas des médecins, ce choix ne nous semble pas pertinent. C'est ce que nous expliquons plus loin dans ce même encadré, en justifiant notre choix de la contrainte (3).

\section{Exogénéité des variables explicatives}

Le modèle (1) est estimé par les moindres carrés ordinaires, ce qui revient à supposer que les variables explicatives sont exogènes. Or, Bolduc et al. (1996) ont montré dans le cas du Québec que les choix de localisation des médecins sont influencés par le niveau de revenu qu'ils peuvent espérer en exerçant dans une région plutôt qu'une autre. Les variables décrivant la localisation géographique des médecins peuvent donc être non exogènes. Trois variables de ce type figurent dans notre modèle: la densité médicale, le type de localisation (pôle urbain, couronnes périurbaines, communes multipolarisées, espace à dominante rurale) et la région d'exercice.

Le problème de non-exogénéité potentielle de certaines variables peut être éludé en transformant le modèle par les différences premières afin d'éliminer l'hétérogénéité non observée figurant dans la perturbation et susceptible d'être corrélée avec les variables de localisation. Mais cette solution empêcherait toute identification des effets cohorte auxquels nous sommes intéressées au premier chef. Pour la même raison, des fixes individuels propres au médecin ne sont pas inclus dans la régression.

Pour examiner l'exogénéité des variables de localisation nous avons mis en œuvre des tests d'Hausman, mais de façon limitée à cause du faible nombre d'instruments disponibles. Un instrument pertinent est une variable exogène (hypothèse validée par un test de Sargan), qui doit expliquer significativement la variable dont on veut tester l'exogénéité, et qui ne doit pas figurer de façon significative dans le modèle principal (le modèle (1)).

II a été possible de réaliser un test d'Hausman pour tester l'exogénéité de la densité d'omnipraticiens. 
Encadré 3 (suite)

Les variables expliquant la demande de soins dans le département peuvent constituer de bons instruments. Deux variables seulement ont un impact significatif sur la densité d'omnipraticiens et sont exclues de la régression principale, c'est-à-dire non significatives dans le modèle (1) : la proportion de femmes dans le département d'exercice du médecin $i$ et le logarithme du revenu moyen des ménages vivant dans le département. Mené avec ces deux instruments, le test d'Hausman ne permet pas de rejeter l'exogénéité de la densité d'omnipraticiens $(p=0,90)$. Par ailleurs, le test de Sargan valide l'exogénéité de ces instruments $(p=0,22)$. Un instrument supplémentaire peut être obtenu en considérant la densité de spécialistes, qui n'est pas significative dans l'équation principale. $\mathrm{Ce}$ nouvel instrument, joint à la proportion de femmes et au revenu des ménages, permet alors de tester, non seulement l'exogénéité de la densité de médecins généralistes mais aussi celle du type de localisation (agrégé en trois catégories : zone urbaine, zone rurale et couronnes). L'exogénéité de ces variables $(p=0,26)$ est validée par ce nouveau test d'Hausman (avec un test de Sargan qui confirme la validité des instruments). La régression de première étape montre que la densité de spécialistes a une influence positive sur la densité de médecins généralistes, suggérant une complémentarité entre l'activité de ces deux catégories de médecins.

Les résultats de ces tests peuvent paraître surprenants. De fait, le nombre d'instruments disponibles étant très restreint, nous ne pouvons pas tester l'exogénéité de toutes les variables de localisation. En particulier, nous ne pouvons pas tester l'exogénéité des dummies régionales, lesquelles figurent dans la liste des variables explicatives du modèle. Les conclusions de nos tests d'Hausman ont donc une portée limitée.

\section{Biais de sélection}

Notre échantillon est non cylindré car il est représentatif des installations en cours de période et des départs en retraite. Mais des médecins peuvent aussi quitter l'échantillon parce qu'ils sortent du champ d'étude, la médecine libérale en secteur 1. Les motifs de leur décision peuvent être en rapport avec le phénomène étudié : nos estimations seraient alors affectées par un biais de sélection. Par exemple, si les médecins d'une cohorte défavorisée partent dans une plus grande proportion, et si les sortants sont ceux qui réussissent le moins bien (qui ont les honoraires les plus bas), l'estimation surestimera l'effet fixe pour cette cohorte.

On observe que les sorties de l'échantillon peuvent être définitives ( $9 \%$ des observations, $17 \%$ des médecins) ou provisoires (6 \% observations, $6 \%$ des médecins). Les raisons de la sortie sont connues de manière très partielle : le passage en secteur 2 concerne $34 \%$ des médecins qui quittent définitivement l'échantillon et $26 \%$ des médecins qui connaissent une interruption provisoire (ils finissent donc par réintégrer le secteur 1 ). Les déménagements concernent $17 \%$ des médecins qui connaissent une interruption provisoire. Les autres raisons, non observées, de sortie définitive ou d'interruption, peuvent être le passage à une activité salariée

(exercice de la médecine ou autre), le décès ou une incapacité de travail. La durée médiane d'une interruption est 2 années, 2,5 années pour un déménagement et 6 années pour un passage en secteur 2 (suivi d'un retour au secteur 1). On observe que la proportion de sorties et d'interruptions est plus élevée pour les cohortes 1978 à 1985 ainsi qu'en début de période (entre 1983 et 1987). On ne trouve pas de différence significative entre les hommes et les femmes médecins concernant la proportion de sorties et leur durée, mais tous les médecins qui sortent ou s'interrompent ont moins d'expérience et des honoraires plus bas.

II est difficile de corriger le biais de sélection par la méthode de Heckman (1979) pour deux raisons : tout d'abord, aucun instrument n'est disponible en dehors des variables du modèle pour expliquer la présence du médecin dans l'échantillon; ensuite les types de sortie et les raisons de sortie sont multiples : il n'est pas pertinent de les formaliser à l'aide d'une unique équation de participation. Nous avons adopté l'approche proposée par Verbeek et Nijman, (1992), qui consiste à ajouter au modèle différentes variables indicatrices du statut du médecin au regard de sa participation. Cette méthode ne corrige évidemment pas le biais de sélection, mais permet de tester son existence par un test de significativité globale des différentes indicatrices.

Le modèle (1) a été estimé sous les contraintes d'identification, en ajoutant cinq dummies signalant l'existence d'une interruption pour passage en secteur 2, pour déménagement, pour une autre raison, ou d'une sortie définitive pour passage en secteur 2 ou pour une autre raison. Ces variables sont significatives (la statistique de Fisher est telle que $p<0,0001$ ), et les coefficients estimés attestent que les médecins qui sortent sont bien ceux qui ont des honoraires plus bas, toutes choses égales par ailleurs (cf. tableau cidessous). II y a donc bien un biais de sélection. Ce dernier est toutefois d'une ampleur très limitée et n'affecte aucunement les profils obtenus pour les divers effets fixes. Les graphiques VI à VIII, qui représentent les effets fixes estimés fournissent tous les résultats obtenus avec et sans les dummies de participation: on constate qu'il n'y a pas de différence. Les résultats changent (très peu) pour d'autres coefficients, comme par exemple celui de la densité $(-0,00263$ au lieu de - 0,00245 ) ou celui de la variable indicatrice du deuxième sexe $(-0,336$ au lieu de - 0,343$)$.

\section{Choix de contrainte pour l'identification du modèle}

L'identification d'effets d'expérience (ou d'âge), de date et de cohorte est un problème maintes fois rencontré dans les études sur données longitudinales. Kessler et Masson (1985), puis Deaton (1997) et Allain (1997) ont formalisé clairement le problème de façon à guider les choix empiriques. Sur des données concernant des individus qui ne connaissent pas d'interruption de carrière, une colinéarité stricte relie les effets de date, de cohorte et d'expérience : $t=c+e$. L'identification des différents effets n'est possible que si l'on ajoute une contrainte supplémentaire à l'ensemble de contraintes (2) (lesquelles permettent d'éviter une colinéarité avec la constante du modèle). 
Encadré 3 (suite)

Nous ne sommes pas dans le cas d'une colinéarité stricte $t=c+e$ car $6 \%$ des observations concernent des médecins ayant connu des interruptions de carrière. Pour ceux-ci, on a $t>c+e$. Le modèle est donc identifiable sans l'ajout d'une contrainte additionnelle, même si $t$ est fortement corrélé avec $c+e$. Cependant, il nous a paru important que les résultats ne reposent pas sur une identification fondée sur les observations minoritaires.

La contrainte additionnelle peut être spécifiée de façons très variées. Par exemple, on peut choisir d'annuler un deuxième effet spécifique pour-au choix-l'expérience, la date, ou la cohorte; on peut aussi imposer l'absence de tendance sur les effets concernant - au choix - l'expérience, la date, ou la cohorte. On peut encore considérer des effets fixes relatifs à des groupes (d'expérience, de date, ou de cohorte). Dans ce cas cependant, il faut être conscient qu'on impose plus de contraintes qu'il n'est nécessaire: une seule contrainte linéaire additionnelle permet l'identification du modèle. L'expérience montre que le choix de la contrainte identifiante influence beaucoup les résultats. II convient donc de justifier rigoureusement le choix effectué.

En pratique, la littérature empirique se concentre sur le choix entre les deux contraintes additionnelles suivantes:

l'absence de tendance sur l'effet cohorte:

$\sum_{c} c * \gamma_{c}=0$ (3) ;

ou l'absence de tendance sur l'effet temporel: $\sum_{t} t * \delta_{t}=0$ (4).

En se référant à des travaux réalisés sur données françaises, on trouve les exemples de Lollivier et Payen

\section{Coefficients estimés des cinq variables indicatrices du statut de participation du médecin}

\begin{tabular}{|l|c|c|}
\hline \multicolumn{1}{|c|}{ Variable } & $\begin{array}{c}\text { Coefficient } \\
\text { estimé }\end{array}$ & Significativité \\
\hline $\begin{array}{l}\text { Interruption pour } \\
\text { passage en secteur 2 }\end{array}$ & $-0,122$ & $\mathrm{P}<0,0001$ \\
\hline $\begin{array}{l}\text { Interruption pour } \\
\text { déménagement }\end{array}$ & $-0,172$ & $\mathrm{P}<0,0001$ \\
\hline $\begin{array}{l}\text { Interruption pour autre } \\
\text { raison }\end{array}$ & $-0,082$ & $\mathrm{P}<0,0001$ \\
\hline $\begin{array}{l}\text { Sortie définitive pour } \\
\text { le secteur 2 }\end{array}$ & $-0,033$ & $\mathrm{P}=0,009$ \\
\hline $\begin{array}{l}\text { Sortie définitive pour } \\
\text { autre raison }\end{array}$ & $-0,213$ & $\mathrm{P}<0,0001$ \\
\hline
\end{tabular}

Lecture: toutes choses égales par ailleurs, les médecins qui sortent provisoirement de l'échantillon pour passer en secteur 2 ont des honoraires inférieurs de $12,2 \%$ à ceux des autres médecins.

Champ: échantillon utilisé pour l'estimation: médecins du secteur 1, installés entre 1970 et 2001 et présents au moins une fois dans la base entre 1983 et 2004.

Source : panel d'omnipraticiens libéraux du SNIR (CNAMTS), 1983-2004.
(1990) qui adoptent (3) et de Bourdallé et Cases (1996), qui retiennent plutôt (4). Dans les deux cas, l'espace de projection est le même : il n'y a pas de fondement théorique à choisir une contrainte plutôt qu'une autre.

Montrons tout d'abord que les résultats sont très sensibles au choix de la contrainte. Pour ne pas multiplier les résultats, nous nous concentrons sur l'effet de l'expérience et sur le choix entre les contraintes (3) et (4). Le graphique A représente les effets de l'expérience estimés avec les trois contraintes envisagées pour l'identification: " pas de tendance sur l'effet cohorte » (3), « pas de tendance sur l'effet temporel » (4) et « groupes de cohortes».

Le profil estimé dépend très fortement du choix entre les contraintes (3) et (4). L'influence des contraintes est facile à interpréter : si on adopte la contrainte (4), qui exclut toute tendance sur l'effet temporel, les revalorisations tarifaires qui ont fait progresser les honoraires des médecins sur la période sont saisies par l'effet de l'expérience. La courbe qui en résulte est fonction croissante de l'expérience car la proportion de médecins avec un haut niveau d'expérience croît avec le temps dans la population médicale comme dans notre échantillon : les médecins avec plus de vingt ans d'expérience représentent $7,5 \%$ de l'échantillon en 1993, $25 \%$ en 1998 et $41 \%$ en 2004. C'est ce type de résultat qui est obtenu par Audric (2006) qui adopte la contrainte (4). On obtiendrait aussi un profil d'honoraires fonction croissante de l'expérience avec une analyse descriptive de l'évolution des honoraires par

\section{Graphique A}

Estimation des effets spécifiques expérience avec trois contraintes différentes

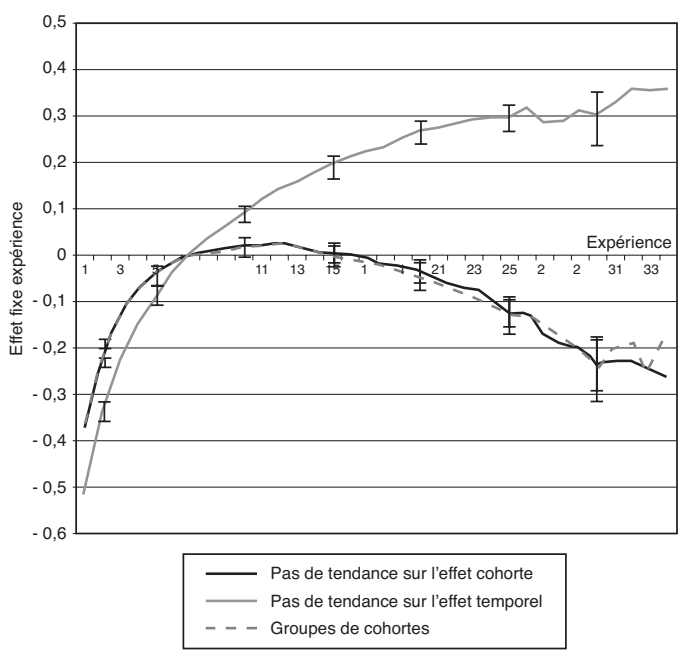

Lecture : effets fixes expérience estimés avec trois contraintes différentes pour l'identification: i) pas de tendance sur l'effet cohorte ; ii) pas de tendance sur l'effet temporel ; iii) groupes de cohortes. Ces effets fixes sont tous exprimés par rapport à une expérience de référence égale à sept ans. Les estimations sont quasi confondues lorsqu'on considère des groupes de cohortes ou la contrainte (i).

Champ: échantillon utilisé pour l'estimation: médecins du secteur 1, installés entre 1970 et 2001 et présents au moins une fois dans la base entre 1983 et 2004.

Source : panel d'omnipraticiens libéraux du SNIR (CNAMTS), 1983-2004. 
Encadré 3 (suite)

cohorte en fonction de l'expérience : de nature univariée, ce type d'analyse ne contrôle pas l'effet temporel. De ce fait, l'effet de l'expérience incorpore tout l'effet des revalorisations tarifaires, ce qui peut donner lieu à des erreurs d'interprétation (voir par exemple LucasGabrielli et Sourty-Le Guellec, 2004).

Pour adopter la contrainte (3), il faut trouver un ou plusieurs résultats empiriques accréditant l'hypothèse d'absence de tendance sur l'effet cohorte. Dans notre cas, le problème est assez simple, puisqu'il nous est possible d'estimer le modèle sans contrainte additionnelle. En effet, les sorties transitoires de l'échantillon font que $6 \%$ des observations sont telles que $t>c+e$ : le modèle est identifiable stricto sensu. Les résultats obtenus par le modèle sans contrainte (autres que (2)) sont satisfaisants. Certes, la forte corrélation entre $t$ et $c+e$ entraîne une perte de précision, les écart-types estimés des coefficients doublant ou triplant selon les cas. Mais les coefficients restent significatifs. On a représenté dans le graphique B les effets cohorte estimés selon trois stratégies possible pour l'identification.

Les effets cohorte estimés avec le modèle sans contrainte additionnelle sont très proches de ceux estimés avec la contrainte (3) : les courbes sont quasiment confondues. II est naturel dans ce cadre de procéder à un test de Fisher. Celui-ci permet de ne pas rejeter la validité de la contrainte (3), avec un risque de première espèce $p=0,84$. En revanche, le test de la contrainte (4) conduit à un rejet sans appel $(p<0,0001)$. Ces résultats suffisent à justifier l'estimation du modèle en

\section{Graphique B \\ Estimation des effets spécifiques cohorte avec trois méthodes d'estimation}

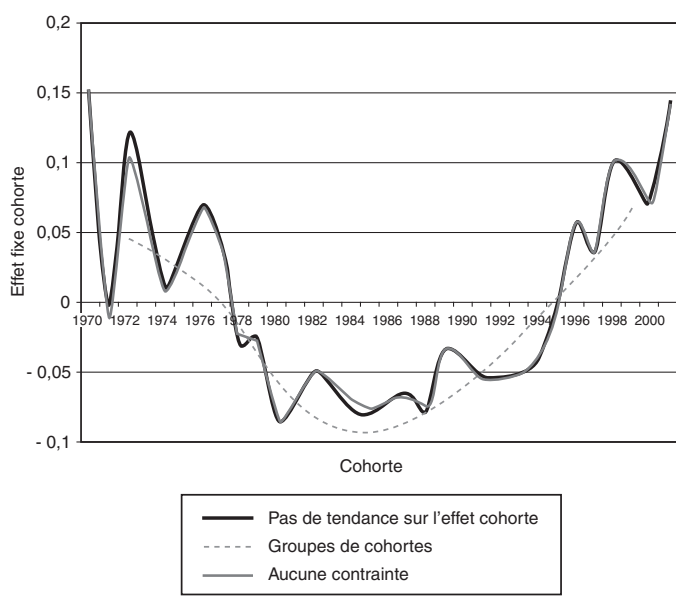

Lecture: effets spécifiques cohorte estimés avec trois contraintes différentes pour l'identification: i) pas de tendance sur l'effet cohorte ; ii) groupes de cohortes ; iii) aucune contrainte additionelle. II n'y a pas de cohorte de référence, mais la somme des effets cohorte est contrainte à être égale à 0 . Les estimations sont très proches, quelle que soit la spécification retenue.

Champ: échantillon utilisé pour l'estimation: médecins du secteur 1, installés entre 1970 et 2001 et présents au moins une fois dans la base entre 1983 et 2004.

Source : panel d'omnipraticiens libéraux du SNIR (CNAMTS), 1983-2004 appliquant la contrainte (3) : celle-ci est validée par le test de Fisher et elle permet de gagner en précision.

Par ailleurs, nous avons considéré un modèle spécifiant des effets fixes pour des groupes de cohortes $g$. Agréger les cohortes par groupes constitue un ensemble de contraintes identifiantes car $t \neq($ groupes de $c)+e$. Comme nous l'avons précisé ci-dessus, un tel modèle est toutefois trop contraignant : une seule contrainte additionnelle est nécessaire. Imposer plus de contraintes augmente les risques de biais. L'estimation des effets cohorte obtenus par la spécification en termes de groupes de cohortes figure aussi dans le graphique $B$ : on obtient une courbe d'allure similaire avec ce qui est obtenu lorsque l'on applique la contrainte (3).

En retournant aux effets de l'expérience (cf. graphique A), on constate que les effets estimés avec la contrainte (3) ne sont pas significativement différents de ceux estimés avec la spécification en termes de groupes de cohortes. Nous n'avons pas représenté les effets de l'expérience estimés par le modèle sans contrainte additionnelle. En effet, leurs estimations sont confondues avec celles des effets estimés sous la contrainte (3).

Cette analyse empirique permet de conclure à l'absence de tendance sur l'effet cohorte. Au-delà de la démarche économétrique, ce résultat est cohérent avec notre connaissance historique de la période étudiée: les tarifs ont été régulièrement relevés par les accords conventionnels entre 1983 et 2004, ce qui disqualifie l'hypothèse d'absence de tendance sur l'effet temporel ; le baby-boom et l'introduction du numerus clausus ont entrainé de grandes fluctuations dans la démographie médicale, ce qui est compatible avec l'absence de tendance sur l'effet cohorte.

\section{Séparabilité des effets fixes}

Une hypothèse implicite du modèle estimé est celle de séparabilité (ou d'additivité) des effets spécifiques. Autrement dit, nous supposons qu'il n'y a pas d'effet croisé expérience-cohorte: le profil de l'expérience est supposé identique pour toutes les cohortes.

Les cohortes de l'échantillon ne recouvrent pas toutes la même plage d'expérience (cf. tableau A de l'encadré 2). Nous avons pu toutefois réaliser quelques estimations à caractère exploratoire sur des paires de cohortes pour examiner le caractère contraignant de cette hypothèse d'absence d'effet croisé. Sur les cohortes 1972 et 1977 la plage de variation de l'expérience est $11-27$ ans : les effets croisés ne sont pas significatifs $(F=1,13 ; p=0,323)$. Pour les cohortes 1977 et 1985 la plage de variation de l'expérience est 6 - 19 ans : les effets croisés sont significatifs $(F=6,10 ; p<0,0001)$. Enfin, pour les cohortes 1972 et 1985 la plage de variation de l'expérience est 11 à 19 ans : les effets croisés sont significatifs $(F=2,65 ; p=0,007)$. On peut donc conclure que pour certaines cohortes l'estimation de la spécification supposant une séparabilité des effets est biaisée. Les effets croisés obtenus sont positifs : les médecins des cohortes défavorables compensent leur mauvaise situation moyenne en ralentissant moins 
l'année, de l'expérience et de la cohorte. Une telle spécification n'est pas identifiable sans l'ajout de contraintes sur les effets. Le choix des contraintes adoptées est décrit et justifié dans l'encadré 3.

Nous avons dû limiter l'analyse économétrique aux cohortes comportant un nombre suffisant de médecins, à savoir les cohortes 1970 à 2001. De ce fait, les estimations sont menées sur 6016 médecins ayant de 1 à 34 ans d'expérience. Trois versions du modèle ont été estimées ; elles diffèrent par la variable expliquée : le logarithme des honoraires, le logarithme de l'activité et enfin le logarithme des revenus (estimés). La construction des revenus est décrite dans l'encadré 2. L'activité est définie comme le nombre annuel de rencontres du médecin avec ses patients, en comptant chaque rencontre pour une, qu'elle soit cotée en C (consultation), en $\mathrm{V}$ (visite), en $\mathrm{K}$ (actes de chirurgie) ou en $\mathrm{Z}$ (actes de radiologie). Comme les tarifs sont fixés, les différences observées entre honoraires et activité sont dues au contenu moyen en actes de chaque rencontre. Chez des généralistes du secteur 1 , les consultations représentent $64 \%$ à $74 \%$ de l'activité. Comme on le voit dans Delattre et Dormont (2005) la structure de l'activité joue toutefois un rôle significatif sur les honoraires : en médiane, on trouve que les honoraires sont majorés de $6 \%$ par rapport à une activité qui serait exclusivement composée de consultations au cabinet. Très quantitatif, l'indicateur " activité » se focalise sur le nombre de rencontres, captant principalement l'information sur la durée du travail du médecin et la taille de sa clientèle.

Le modèle est estimé par les moindres carrés ordinaires sous contrainte. Les résultats obtenus pour le logarithme des honoraires sont présentés dans le tableau 1, à l'exception des estimations des effets fixes, présentées dans les graphiques VI à VIII. Les graphiques IX à XI permettent de comparer les estimations obtenues sur les honoraires et les revenus.

\section{Les femmes médecins perçoivent des honoraires plus bas}

Les femmes médecins ont, toutes choses égales par ailleurs, des honoraires inférieurs de $34 \%$ à ceux de leurs homologues masculins (cf. tableau 1). Ces différences d'honoraires s'expliquent entièrement par le nombre de rencontres entre médecin et patient : sur la spécification expliquant l'activité, on trouve que celle des femmes est inférieure de $33 \%$ à celles des hommes (7). Pour les médecins, on trouve ainsi un écart de rémunération entre hommes et femmes supérieur à l'écart moyen observé au niveau global pour les salariés. Le contexte est cependant différent, puisque dans le secteur 1 , les femmes médecins ne peuvent subir de discrimination sur le tarif de la consultation : toute la différence est due à une moindre activité. Ce résultat confirme des analyses plus descriptives effectuées par Fivaz et Le Laidier (2001) sur les généralistes français : en moyenne, les femmes médecins s'absentent un jour de plus par semaine que les hommes et réalisent moins d'actes par jour.

Cette moindre activité des femmes reste à analyser : exprime-t-elle une "préférence » plus marquée des femmes en faveur du loisir? Ou bien les femmes souffrent-elles d'une discrimination de la part des patients? Ou encore leur durée de consultation est-elle plus longue? Pour les États-Unis, Rizzo et Zeckhauser (2007) ont étudié ces questions à l'aide de données concernant de jeunes médecins. Ils obtiennent le même écart de revenu entre les hommes et les femmes : $33 \%$ en 1990 . Le contexte institutionnel américain est assez différent, car les tarifs y sont souvent déterminés par le médecin, ce qui peut contribuer à accentuer l'impact d'éventuels comportements discriminatoires (8). Explorant

7. Pour alléger la présentation, nous ne publions pas ici le détail des résultats obtenus pour les spécifications expliquant le logarithme de l'activité et celui des revenus.

8. Si la fonction de demande de soins s'adressant aux femmes médecins est plus faible, le tarif d'équilibre de la consultation devrait être plus faible pour les femmes médecins que pour les hommes médecins.

\section{Encadré 3 (fin)}

leur activité avec l'expérience. En revanche, ce profi plus plat va de pair avec une constante spécifique à la cohorte encore plus défavorable que celle estimée dans le modèle avec séparabilité, qui semble sousestimer les écarts entre cohortes.

Un résultat important de notre étude est le profil obtenu pour l'expérience : les médecins diminuent fortement leur activité après 12 ans d'expérience et ceci peut être interprété comme l'expression d'une préférence pour le loisir. Nos résultats sur les effets croisés sont très partiels, plutôt instables et difficile à interpréter. Ils suggèrent toutefois que cette latitude serait plus limitée pour les médecins des cohortes défavorables: ceux-ci auraient perçu trop peu d'honoraires en début de carrière pour pouvoir alléger comme leurs collègues plus favorisés leur activité au-delà de 12 ans d'exercice. 
les causes possibles d'un tel « gender gap », ces auteurs trouvent que l'écart est presque entièrement expliqué par des différences dans les préférences. Les femmes-médecins ont un revenu

Tableau 1

Estimation de la fonction de gain pour les omnipraticiens du secteur 1

\begin{tabular}{|c|c|c|}
\hline Variable & Coefficient & Écart-type \\
\hline $\begin{array}{l}\text { Sexe } \\
\text { Homme } \\
\text { Femme }\end{array}$ & $\begin{array}{c}\text { Réf. } \\
-0,3429\left(^{\star \star \star}\right) \\
\end{array}$ & $\begin{array}{c}\text { Réf. } \\
0,0041\end{array}$ \\
\hline $\begin{array}{l}\text { Mode d'exercice particulier } \\
\text { (MEP) }\end{array}$ & $-0,0650\left(^{\star \star \star}\right)$ & 0,0069 \\
\hline $\begin{array}{l}\text { Durée entre la thèse et l'ins- } \\
\text { tallation en libéral (en années) }\end{array}$ & $-0,0224\left(^{\star \star \star}\right)$ & 0,0007 \\
\hline $\begin{array}{l}\text { Durée du travail (médecine } \\
\text { libérale) } \\
\text { À temps plein } \\
\text { À temps partiel } \\
\text { Avec temps partiel hospitalier }\end{array}$ & $\begin{array}{c}\text { Réf. } \\
-0,0524\left(^{(\star \star)}\right. \\
0,0019\end{array}$ & $\begin{array}{c}\text { Réf. } \\
0,0045 \\
0,0024\end{array}$ \\
\hline $\begin{array}{l}\text { Catégorie d'aire d'urbaine } \\
\text { Pôle urbain } \\
\text { Couronne périurbaine } \\
\text { Commune multipolarisée } \\
\text { Espace à dominante rurale }\end{array}$ & $\begin{array}{c}\text { Réf. } \\
0,0903\left(^{\star \star \star}\right) \\
0,1121\left(^{\star \star \star}\right) \\
0,1452\left(^{\star \star *}\right)\end{array}$ & $\begin{array}{c}\text { Réf. } \\
0,0049 \\
0,0079 \\
0,0044\end{array}$ \\
\hline $\begin{array}{l}\text { Densité d'omnipraticiens } \\
\text { dans le département d'exer- } \\
\text { cice }\end{array}$ & $-0,0025\left(^{\star \star \star}\right)$ & 0,0002 \\
\hline $\begin{array}{l}\text { Densité de spécialistes dans } \\
\text { le département d'exercice }\end{array}$ & $-7.78^{\star} 10-6$ & 0,00009 \\
\hline $\begin{array}{l}\text { Région d'exercice de la } \\
\text { médecine } \\
\text { Île-de-France } \\
\text { Rhône-Alpes } \\
\text { Picardie } \\
\text { Auvergne } \\
\text { PACA } \\
\text { Champagne-Ardenne } \\
\text { Midi-Pyrénées } \\
\text { Languedoc Roussillon } \\
\text { Basse Normandie } \\
\text { Poitou Charente } \\
\text { Centre } \\
\text { Limousin } \\
\text { Corse } \\
\text { Bourgogne } \\
\text { Bretagne } \\
\text { Aquitaine } \\
\text { Franche Comté } \\
\text { Haute Normandie } \\
\text { Pays de la Loire } \\
\text { Lorraine } \\
\text { Nord } \\
\text { Alsace }\end{array}$ & 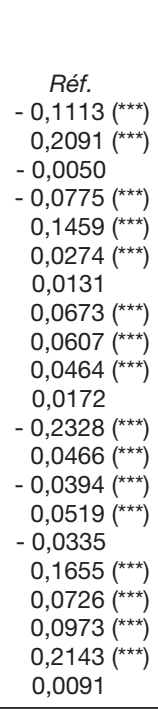 & $\begin{array}{c}\text { Réf. } \\
0,0077 \\
0,0115 \\
0,0126 \\
0,0106 \\
0,0124 \\
0,0107 \\
0,0101 \\
0,0117 \\
0,0101 \\
0,0094 \\
0,0125 \\
0,0237 \\
0,0104 \\
0,0084 \\
0,0084 \\
0,0129 \\
0,0105 \\
0,0092 \\
0,0097 \\
0,0093 \\
0,0093\end{array}$ \\
\hline $\mathrm{R}^{2}$ & 0,2755 & \\
\hline Fisher & 269,56 & \\
\hline Taille de l'échantillon & 81691 & \\
\hline
\end{tabular}

Lecture : la variable expliquée est le logarithme des honoraires. Par rapport aux médecins de la région de référence (Ille-deFrance), les médecins de la région Rhône-Alpes ont, toutes choses égales par ailleurs, des honoraires inférieurs de $11,1 \%$. ${ }^{* * *}$ : Significatif au seuil de $1 \% ;{ }^{* *}$ : significatif au seuil de $5 \%$ * : significatif au seuil de $10 \%$.

Champ : échantillon utilisé pour l'estimation : médecins du secteur 1, installés entre 1970 et 2001 et présents au moins une fois dans la base entre 1983 et 2004

Source: panel d'omnipraticiens libéraux du SNIR (CNAMTS), 1983-2004. de référence (9) inférieur de $26 \%$ au revenu de référence de leurs collègues masculins. En outre, ces derniers réagissent activement lorsque leur revenu passe en dessous de leur niveau de référence : sans augmenter leur durée totale de travail, ils raccourcissent la durée de consultation et modifient la structure des soins fournis en faveur d'une plus grande proportion de procédures plus rémunératrices.

Les résultats montrent également que les médecins ayant un mode d'exercice particulier (homéopathie, acupuncture, etc.) ont des honoraires inférieurs de $6,5 \%$ à ceux des autres médecins. Cette différence est due à une moindre activité (on obtient - $32 \%$ pour le coefficient de la variable indicatrice MEP dans l'équation expliquant le nombre de rencontres médecin-patient), fortement compensée par un plus grand contenu en actes de la rencontre, permettant à celle-ci d'être plus rémunératrice.

\section{La localisation influence beaucoup les honoraires}

La densité d'omnipraticiens dans le département d'exercice joue négativement sur les honoraires du médecin : une augmentation de la densité de 10 (passage de 100 à 110 médecins pour 100000 habitants par exemple) conduit à une diminution des honoraires de 2,5\%. L'impact est encore plus marqué pour l'activité : une augmentation de la densité de 10 conduit à une diminution du nombre de rencontres médecin-patient de $3,5 \%$. Cette diminution est due à la baisse de la demande qui s'adresse à chaque médecin quand des médecins en plus grand nombre se partagent la même quantité de patients. Dans le secteur 1 , cette baisse se répercute à l'identique sur les honoraires, à moins d'une augmentation du contenu moyen en actes de chaque rencontre. Celle-ci apparaît clairement dans le fait que le choc sur les honoraires est atténué : $-2,5 \%$ au lieu de - 3,5\% (10). Les deux coefficients ici obtenus traduisent un impact non négligeable de la localisation, d'autant plus qu'il s'agit d'un effet différentiel qui s'ajoute à la constante régionale (11), laquelle incorpore

9. Recueillie par enquête, cette variable désigne un revenu qui semble correct à la personne interrogée, compte tenu de son temps de travail et de son investissement dans des études longues et coûteuses (pour les États-Unis).

10. C'est une des modalités de la demande induite en France (Delattre et Dormont, 2003). À défaut de pouvoir influencer le retour du patient pour des consultations additionnelles, le médecin peut toujours augmenter le nombre d'actes par rencontre.

11. Dans la terminologie de l'économétrie des données de panel, on dirait qu'il s'agit d'un coefficient estimé dans la dimension intra-régionale (" within region »). 
aussi un effet négatif de la densité. En revanche, la densité de spécialistes n'a pas d'effet significatif : le partage des patients s'effectue « entre omnipraticiens $\gg$.

L'effet négatif de la densité sur les honoraires apparaît aussi dans les constantes régionales estimées (cf. graphique V). L'impact est d'une intensité variable, plus marquée pour les régions de faible densité. Plus précisément, le « malus » associé au fait d'être dans une région à densité très élevée (comme en PACA, où il s'élève à $-7,6 \%$ ) est nettement inférieur au «bonus» lié au fait d'être dans une région à faible densité (comme en Picardie ou dans le Nord, où il s'élève à $+21 \%$, ou encore en Champagne Ardennes où il est de 14,6\%).

De façon générale, les effets fixes régionaux captent l'influence sur les honoraires des « aménités », pour reprendre la terminologie de l'éco- nomie spatiale. Il s'agit de l'agrément apporté par le climat, la qualité de vie et les équipements collectifs caractérisant un territoire. Dans ce cadre, les différences d'honoraires estimées par les effets fixes entre, par exemple, le Nord et la région PACA, peuvent s'interpréter à la fois du côté de l'offre et de la demande. Du point de vue de la demande, le nombre de patients potentiels est plus faible en PACA, où la densité est élevée, que dans le Nord, où elle est faible. En ce qui concerne l'offre, les écarts d'honoraires semble refléter les différences d'utilités attendues par les médecins lorsqu'ils choisissent de s'installer (Bolduc et al., 1996). Autrement dit, les médecins qui choisissent de s'installer en PACA acceptent de gagner en moyenne $8 \%$ de moins, car dans cette région ils travailleront moins (auront moins de patients) et profiteront d'un climat plus ensoleillé. A contrario, il semble qu'un supplément d'honoraires d'au moins $20 \%$ soit nécessaire pour compenser le surcroît

Graphique V

Densité moyenne par région et coefficient associé (référence : Île-de-France)

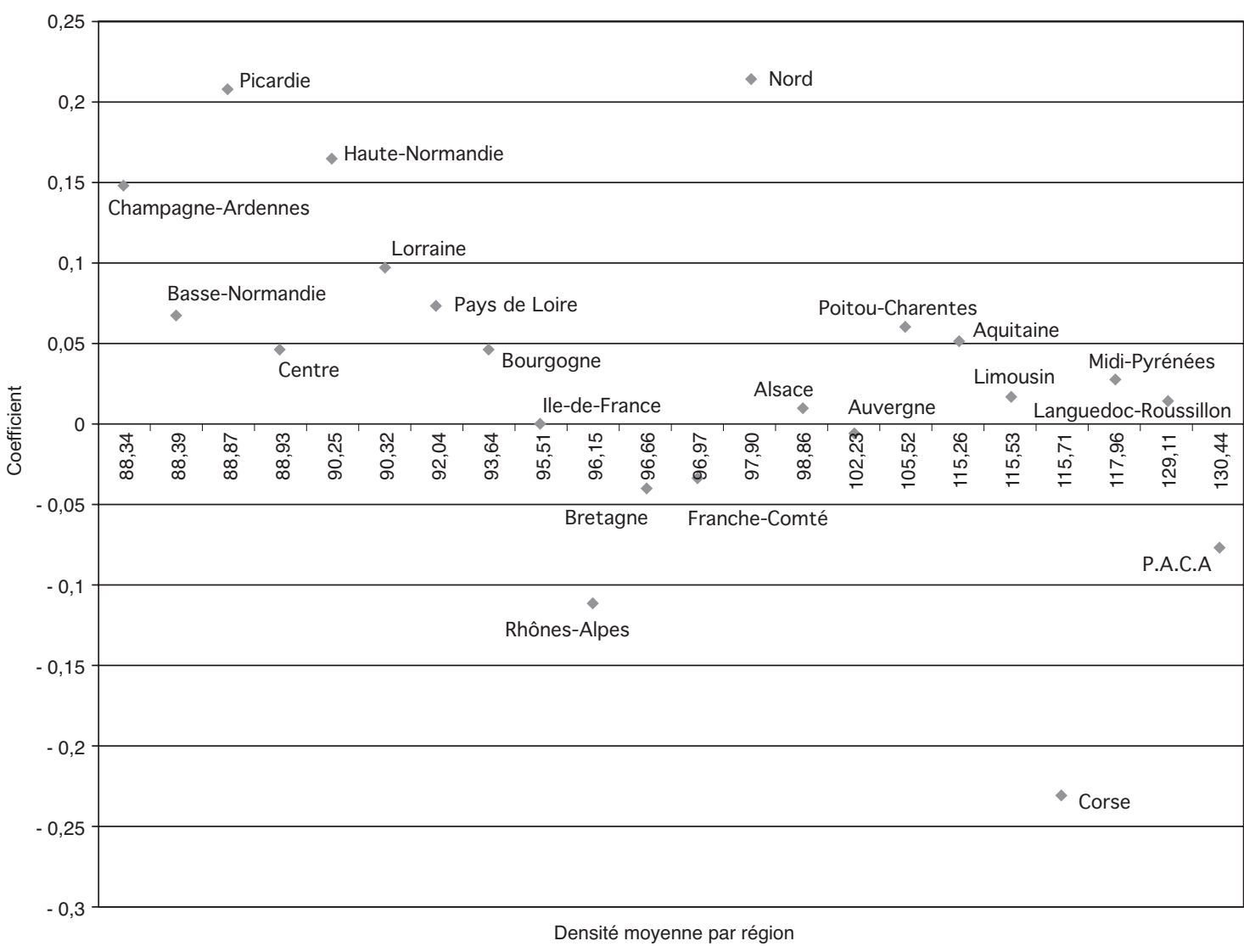

Lecture : les points représentés ont pour ordonnée l'effet fixe régional estimé par le modèle (1) (coefficients présentés dans le tableau 1) et pour abscisse la densité moyenne de la région concernée. L'effet fixe régional mesure l'écart moyen entre les honoraires de la région concernée et ceux de l'lle de France, estimé toutes choses égales par ailleurs.

Par exemple, par rapport aux médecins de la région de référence (Ille-de-France), les médecins de la région Rhône-Alpes ont, toutes choses égales par ailleurs, des honoraires inférieurs de $11,2 \%$ en moyenne.

Champ : Il est défini par l'échantillon sur lequel l'estimation est réalisée : médecins du secteur 1, installés entre 1970 et 2001 et présents au moins une fois dans la base entre 1983 et 2004.

Source : panel d'omnipraticiens libéraux du SNIR (CNAMTS), 1983-2004 et estimations du tableau 1. 
de travail et le climat plus rude qui attendent le médecin qui s'installe dans la région Nord.

Enfin, les estimations montrent que, par rapport à une installation dans un pôle urbain, il y a un intérêt financier à se localiser ailleurs, en couronne périurbaine $(+9 \%)$, dans une commune multipolarisée $(+11,2 \%)$ et surtout dans un espace à dominante rurale $(+14,5 \%)$.

Nous l'avons vu, le contexte actuel est marqué par le développement de mesures financières encourageant l'installation dans les zones déficitaires en médecins. Ces différents résultats sont obtenus sur des années non concernées par les majorations de tarifs ni par les exonérations fiscales introduites à partir de 2004. Ils montrent qu'existent déjà, indépendamment de toute mesure spécifique, de sérieux avantages financiers à l'installation dans les zones déficitaires. Ces avantages sont toutefois associés à une charge de travail plus importante.

\section{L'effet de l'expérience : les médecins généralistes ont un profil de carrière très différent de celui des salariés}

Notre estimation permet de mesurer l'effet de l'expérience sur les honoraires du médecin en contrôlant sa localisation régionale, la densité, son sexe, son mode d'exercice, l'année d'observation et sa cohorte (cf. graphique VI). On obtient une courbe en forme de U renversé : la phase de constitution de clientèle en début de carrière est associée à une progression spectaculaire des honoraires. À partir de 12 années d'expérience, l'activité et les honoraires décroissent lentement, puis plus rapidement après 20 ans d'expérience jusqu'à la cessation d'activité.

L'effet de l'expérience ici estimé n'est pas un effet brut mais est évalué toutes choses égales par ailleurs. Le profil obtenu ne signifie pas nécessairement que les médecins réduisent leur activité après 12 années de carrière : d'autres effets, comme par exemple les effets temporels, interviennent pour déterminer leur niveau d'activité.

Par rapport aux formes connues de l'effet de l'expérience sur les salaires, le profil estimé se distingue par les traits suivants :

- une progression plus forte en début de carrière. Entre la première année et l'expérience de référence (7 ans (12)), il y a une augmentation des honoraires de $37 \%$. Cette forte croissance correspond à la phase de constitution de sa clientèle par le médecin ;

- l'absence de phase en plateau ;

- la diminution après le maximum obtenu avec 12 ans d'expérience. À 25 ans d'expérience la baisse est en moyenne de - $12 \%$ par rapport à la référence ( 7 ans). Elle atteint - $24 \%$ au bout de 30 ans d'expérience.

Le profil de carrière obtenu est robuste à une estimation séparée du modèle pour les hommes et les femmes: entre 0 et 30 années d'expérience par exemple, les effets fixes expérience estimés pour les femmes sont très corrélés avec ceux estimés pour les hommes (le coefficient de corrélation est égal à 0,934$)$.

Par comparaison, les profils de carrière obtenus pour les salariés sont beaucoup moins contrastés (Koubi, 2003b). Tout se passe donc comme si les médecins utilisaient la latitude dont ils disposent grâce à l'exercice d'une profession libérale pour moduler davantage leur activité au cours de leur vie professionnelle que les salariés (13).

12. Des estimations préalables ont montré que c'est le nombre d'années nécessaires à un médecin pour constituer sa clientèle (Delattre et Dormont, 2003).

13. Les différences ici relevées méritent d'être confirmées par une étude sur les salariés avec des méthodes identiques aux nôtres, dans la mesure où les résultats obtenus sur les salariés par Koubi (2003b) découlent de spécifications différentes.

\section{Graphique VI}

Estimation des effets spécifiques expérience pour le logarithme des honoraires

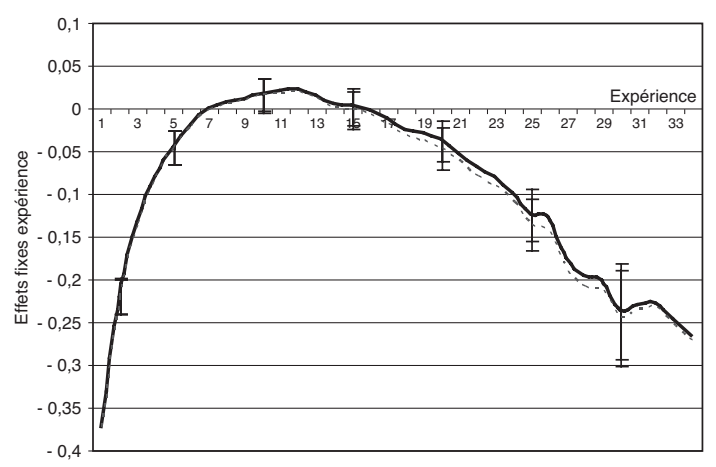

Sans indicatrice de participatio

Avec indicatrices de participation

Lecture : les segments verticaux représentent des intervalles de confiance à $95 \%$ associés à certains effets fixes. Ces effets fixes sont exprimés par rapport à une expérience de référence égale à sept ans. À 25 ans d'expérience, les médecins perçoivent, toutes choses égales par ailleurs, des honoraires inférieurs de $12 \%$ à ceux des médecins qui ont sept ans d'expérience.

Champ : échantillon utilisé pour l'estimation : médecins du secteur 1, installés entre 1970 et 2001 et présents au moins une fois dans la base entre 1983 et 2004

Source: panel d'omnipraticiens libéraux du SNIR (CNAMTS), 1983-2004. 
L'intérêt du profil obtenu pour les médecins réside dans l'expression des préférences qu'il révèle, alors que les salariés ont peu de latitude dans les choix d'allocation de leur temps de travail au cours de leur carrière. Il est possible que le médecin moyen travaille, au total, autant ou plus que le salarié moyen. Mais il préfère concentrer son effort sur les 12 premières années de son existence professionnelle, quitte à alléger ensuite progressivement sa charge de travail. Ce début de carrière peut être associé à un effort d'investissement matériel et à la constitution d'une réputation. La phase de décroissance observée par la suite est-elle choisie, et donc révélatrice d'une préférence pour le loisir? Ou bien est-elle subie ? Une baisse de la demande adressée au médecin parce qu'il serait plus âgé est peu vraisemblable : avec 13 ans d'expérience, celui-ci aurait environ 43 ans. L'hypothèse d'un épuisement professionnel est évoquée par certaines associations de médecins (14).

Pour les salariés, le profil de carrière peut être défini par l'évolution de la productivité individuelle associée à l'expérience avec une perte potentielle de productivité avec l'âge (non confirmée par Aubert et Crépon, 2003). Il peut difficilement être influencé par la durée du travail, car celle-ci est plutôt contrainte et connaît peu de variation avec l'expérience. En revanche, il peut être défini par l'employeur en vue d'instaurer un système de paiement incitatif sur le cycle d'activité (Lazear, 1981). Sur la base de ce raisonnement, Lazear et Moore (1984) prédisent que le profil de carrière des salariés devrait croître plus fortement avec l'expérience que celui des professions libérales, dans la mesure où celles-ci peuvent se passer d'incitation à la productivité. Nos résultats confirment cette hypothèse.

\section{Les effets de date : le rôle des revalorisations tarifaires}

Les effets fixes année relatifs aux honoraires connaissent par rapport à l'année de référence 1983 une croissance forte et régulière (cf. graphique VII), signalant une importante progression du pouvoir d'achat des honoraires des omnipraticiens du secteur 1 au cours de la période (15). En 2004, l'effet fixe s'élève à 0,408 , ce qui équivaut sur les 21 années écoulées à un taux de croissance annuel moyen de $1,6 \%$ (16). On observe que la croissance des honoraires résulte en partie d'une augmentation continue de l'activité au cours de la période, inséparable d'une charge de travail accrue : l'effet fixe temporel pour l'activité est égal à 0,176 en 2004 , ce qui correspond à un taux de croissance annuel moyen de $0,8 \%$.

L'écart entre les courbes des effets fixes obtenus pour les honoraires et l'activité s'explique par les impacts combinés des revalorisations tarifaires et des changements dans la structure de l'activité des médecins. Les principales revalorisations tarifaires sont intervenues en 1988, 1995, 1998, 2002 et 2003. Leur effet sur la croissance des honoraires est indéniable. Par ailleurs, la structure de l'activité des médecins a beaucoup évolué sur la période étudiée : entre

14. Une enquête réalisée par l'Union Régionale des Médecins Libéraux d'île-de-France cherche à identifier les phénomènes de "burn out » des médecins de cette région. Malheureusement, le traitement statistique des résultats ne gère pas les biais de sélection vraisemblables, compte tenu de réponses épistolaires sur le mode du volontariat. $53 \%$ des répondants sont concernés par un tel «burn out ». Les facteurs favorisants sont l'appartenance au secteur 1, la pratique de la médecine générale et un âge compris entre 45 et 50 ans (URML île-de-France, 2007).

15. Ce sont en effet les honoraires déflatés de l'indice des prix à la consommation qui sont considérés.

16. À titre de comparaison, le salaire réel brut moyen aura progressé sur la même période de 0,6\% par an. Cette comparaison est cependant d'une pertinence limitée, puisqu'il faudrait comparer les salaires super bruts d'une part, et les revenus réels des médecins, corrigés des exonérations de charges sociales dont ils bénéficient pour leur propre compte du fait de leur appartenance au secteur 1

\section{Graphique VII}

Estimation des effets spécifiques année pour le logarithme des honoraires et de l'activité

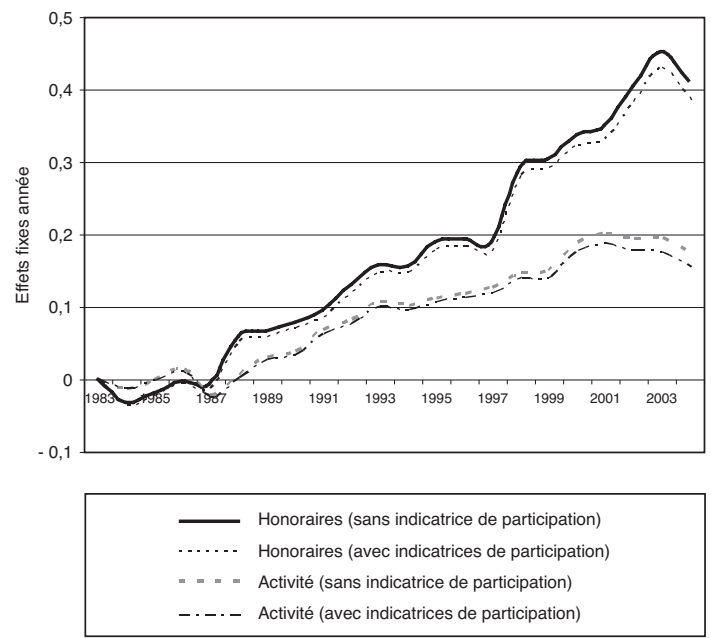

Lecture : les effets fixes année sont estimés sur l'équation expliquant les honoraires d'une part et l'équation expliquant l'activité du médecin, d'autre part. L'activité est définie par le nombre de rencontres médecin patient. L'année de référence est 1983, première année de la période. En 2004, les médecins perçoivent, toutes choses égales par ailleurs, des honoraires supérieurs de 40,8\% à ceux des médecins exerçant en 1983.

Champ : médecins du secteur 1, installés entre 1970 et 2001 et présents au moins une fois dans la base entre 1983 et 2004. Source: Panel d'omnipraticiens libéraux du SNIR (CNAMTS), 1983-2004. 
1983 et 2004, la proportion de visites à domicile est passée de $35 \%$ à $15 \%$ et celle des consultations de $58 \%$ à $82 \%$. Ce changement de structure en faveur des consultations qui sont moins rémunératrices a dû peser négativement sur les honoraires.

Une rapide simulation (17) montre que l'impact négatif du changement de structure est suffisamment important pour annuler en partie la progression due à la croissance de l'activité. En conséquence, la progression des honoraires est due pour l'essentiel à l'effet pur des revalorisations.

\section{Les effets de la cohorte : les générations nombreuses sont défavorisées}

Les effets de la cohorte sur les honoraires des médecins sont d'une ampleur considérable : leur plage de variation atteint 20 points (cf. graphique VIII) (18).

L'effet cohorte est positif pour les cohortes antérieures à 1978, avant de diminuer fortement, puis de se redresser à la fin des années 1980 . Le minimum est atteint pour les cohortes 1980 à 1987. Par exemple, les omnipraticiens installés en 1985 gagnent, toutes choses égales par

\section{Graphique VIII \\ Estimation des effets spécifiques cohorte pour le logarithme des honoraires}

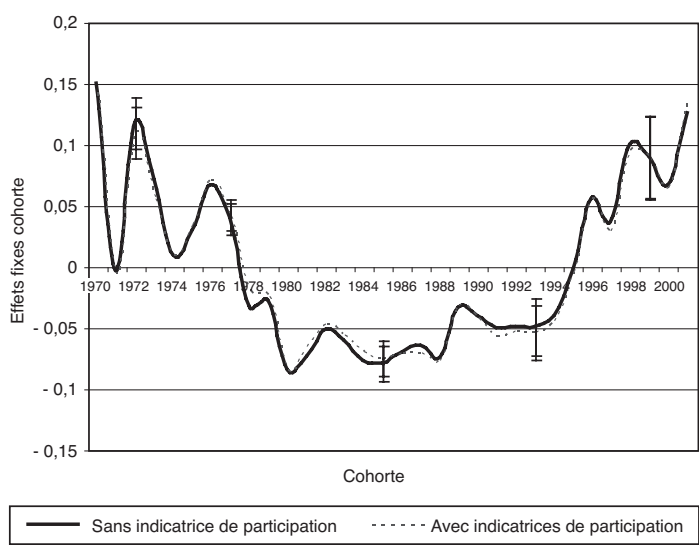

Lecture : les segments verticaux représentent des intervalles de confiance à $95 \%$ associés à certains effets fixes. II n'y a pas de cohorte de référence, mais la somme des effets cohorte est contrainte à être égale à 0 . L'interprétation des résultats est réalisée en étudiant les contrastes entre cohortes considérées par paires. Par exemple toutes choses égales par ailleurs, les médecins installés en 1999 ont des honoraires supérieurs de 16,8 \% à ceux des médecins de la cohorte 1985

Champ : échantillon utilisé pour l'estimation : médecins du sec teur 1, installés entre 1970 et 2001 et présents au moins une fois dans la base entre 1983 et 2004

Source: panel d'omnipraticiens libéraux du SNIR (CNAMTS), 1983-2004 ailleurs, 19,6\% de moins que ceux installés en 1972. Par la suite, la situation se redresse pour les médecins installés dans les années 1990. Toutes choses égales par ailleurs, les médecins installés en 1999 ont des honoraires supérieurs de $16,8 \%$ à ceux des médecins de la cohorte 1985. Le lien entre l'effet cohorte estimé et la régulation de la démographie médicale sera examiné plus loin.

Différentes variantes de l'estimation ont permis de vérifier sa robustesse : en définissant la cohorte par l'année de thèse et non la date d'installation en libéral (19), on obtient un effet cohorte très corrélé avec l'effet initialement estimé (coefficient de corrélation égal à 0,92 ). En restreignant l'estimation aux médecins libéraux à temps complet, ou aux médecins qui n'ont pas de mode d'exercice particulier, on obtient encore un effet très proche (coefficients de corrélation égaux à 0,99 ). C'est à nouveau le cas en croisant l'effet cohorte avec la durée séparant la thèse de l'installation.

\section{Des résultats similaires sur les honoraires et les revenus}

Les revenus n'ont pu être estimés que sur la période 1993-2004 (cf. encadré 2). Pour comparer les résultats obtenus sur les honoraires et les revenus, nous avons dû estimer à nouveau les différents modèles sur cette période plus restreinte.

L'effet de l'expérience se caractérise par une décroissance plus forte du profil en fin de carrière pour les revenus que pour les honoraires (cf. graphique IX). Comme la différence entre ces deux indicateurs de rémunération est due

17. En simplifiant les données pour ne retenir que les consultations et les visites (qui représentent entre $93 \%$ et $97 \%$ de l'activité des médecins selon l'année), nous avons procédé à une rapide simulation pour évaluer l'impact des différentes évolutions sur la progression des honoraires entre 1983 et 2004. Sur ces données modifiées, nous trouvons que la progression de l'activité correspond à une croissance annuelle moyenne de $1,3 \%$ par an, compensée en partie par les changements sur la structure de l'activité, qui diminuent les honoraires de $1 \%$ par an en moyenne. L'essentiel de la progression est alors dû à l'effet pur des revalorisations, qui jouent à hauteur de 1,2\% par an en moyenne.

18. L'effet cohorte connait des fluctuations assez amples avant 1977. Ce résultat curieux est robuste: on obtient les mêmes effets en estimant le modèle avec les indicateurs de présence dans l'échantillon (voir la courbe en pointillés dans le graphique VIII) ou en calculant la médiane, par cohorte, des résidus de l'estimation du modèle (1) de l'encadré 3 sans les variables de cohorte.

19. En effet, les cohortes les plus récentes ont connu un allongement de la durée de leurs études et une augmentation du délai séparant la thèse et l'installation. Ceci pourrait modifier l'estimation de l'effet cohorte tel que nous l'avons défini. 
aux écarts de taux de charge, ce résultat s'explique par l'existence de charges fixes (loyer, secrétariat, etc.) dans un contexte de diminution de l'activité et donc des honoraires. Le même phénomène s'observe en début de carrière, avec la même interprétation : à niveau d'activité faible, taux de charge élevé.

Les effets temporels montrent que les revenus progressent plus vite que les honoraires (cf. graphique $\mathrm{X}$ ) : le rythme des revalorisations tarifaires dépasse nettement le rythme d'évolution des

\section{Graphique IX \\ Estimation des effets spécifiques expérience pour le logarithme des honoraires et des revenus}

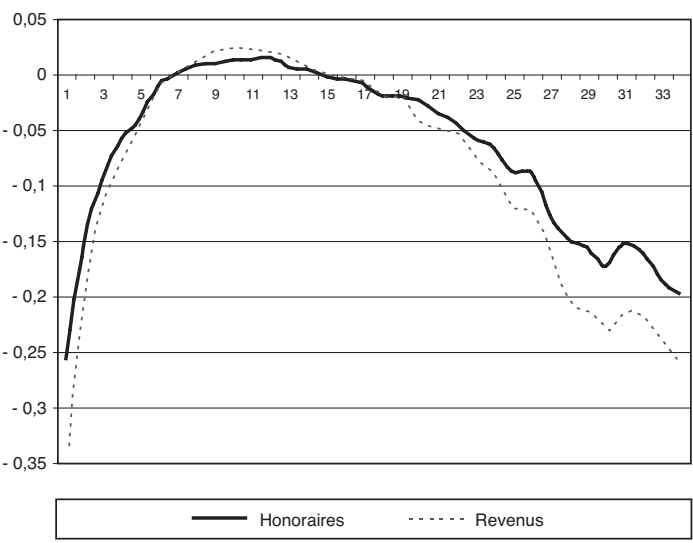

Lecture : l'expérience de référence est égale à sept ans.

Champ : échantillon utilisé pour l'estimation: médecins du sec teur 1, installés entre 1970 et 2001 et présents au moins une fois dans la base entre 1993 et 2004.

Source : panel d'omnipraticiens libéraux du SNIR (CNAMTS) et BNC (DGI/INSEE/DREES), 1993-2004.

\section{Graphique X}

Estimation des effets spécifiques année pour le logarithme des honoraires et des revenus

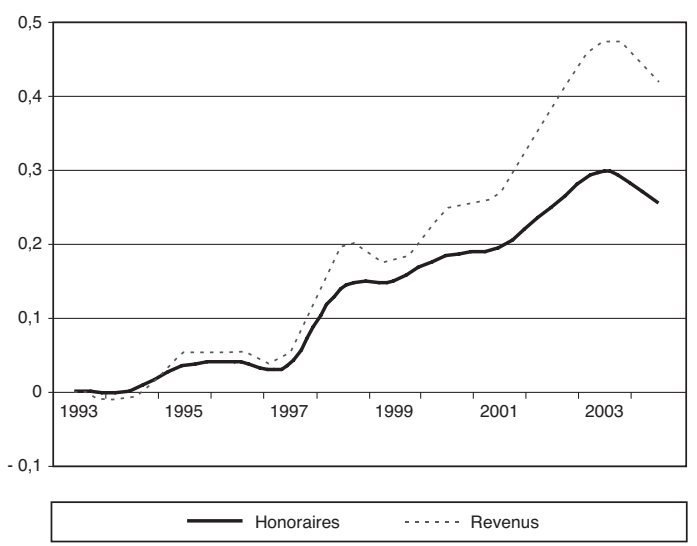

Lecture : l'année de référence est 1993.

Champ : échantillon utilisé pour l'estimation : médecins du secteur 1 , installés entre 1970 et 2001 et présents au moins une fois dans la base entre 1993 et 2004.

Source : panel d'omnipraticiens libéraux du SNIR (CNAMTS) et BNC (DGI/INSEE/DREES), 1993-2004. prix des biens et des services qui déterminent les charges. On retrouve ici le résultat mentionné ci-dessus : sur la période étudiée, la valeur des revenus des médecins, corrigée de l'inflation, a progressé plus rapidement que les revenus réels de l'ensemble des salariés.

Par ailleurs, les effets cohorte ne présentent pas de profils différents pour les honoraires et les revenus (graphique XI) : il n'y pas d'effet cohorte sur les taux de charges.

\section{Comprendre l'effet cohorte}

$\mathbf{L}$ 'effet cohorte mesure les différences moyennes d'honoraires entre médecins ayant débuté leur activité à des dates différentes. Les médecins d'une même cohorte ont des caractéristiques communes : ils appartiennent à la même " génération », avec probablement des préférences similaires concernant le loisir et le style de pratique ; ils ont connu le même numerus clausus et donc le même taux de sélection en fin de première année. Enfin, ils ont débuté leur carrière la même année, partageant un même contexte démographique (20).

20. La densité médicale par département au moment de l'installation est une donnée exogène pour le médecin lorsqu'il procède à son choix de localisation.

\section{Graphique XI}

Estimation des effets spécifiques cohorte pour le logarithme des honoraires et des revenus

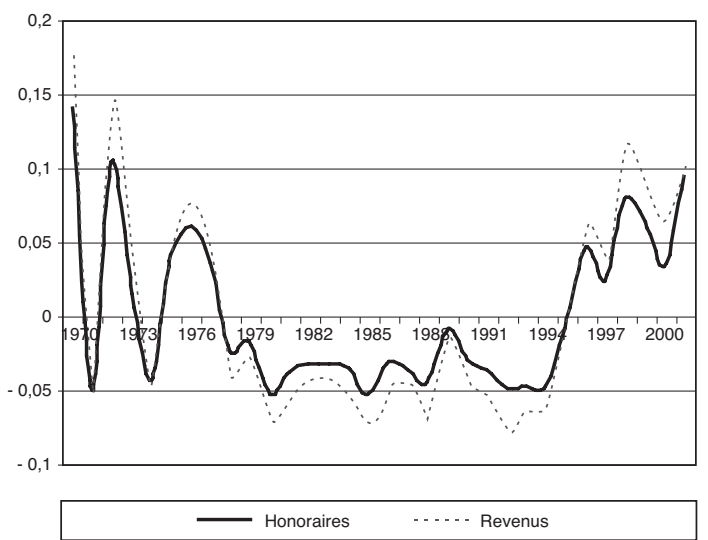

Lecture : il n'y a pas de cohorte de référence, mais la somme des effets cohorte est contrainte à être égale à 0 .

Champ : échantillon utilisé pour l'estimation : médecins du secteur 1, installés entre 1970 et 2001 et présents au moins une fois dans la base entre 1993 et 2004

Source : panel d'omnipraticiens libéraux du SNIR (CNAMTS) et BNC (DGI/INSEE/DREES), 1993-2004. 


\section{Le contexte démographique du début de carrière}

Pour interpréter l'effet cohorte, nous avons remplacé celui-ci dans la spécification (1) (modèle noté (1) dans l'encadré 3) par une fonction linéaire-quadratique de trois variables caractérisant la démographie médicale à la date d'installation: la valeur du numerus clausus régional neuf ans avant l'installation, la valeur de la densité médicale départementale au moment de l'installation et enfin la variation nationale du nombre de médecins au cours de l'année d'installation (le solde des entrants et des sortants). L'estimation de cette fonction linéaire quadratique de trois variables démographiques est représentée sur le graphique XII, sur lequel figure également l'effet cohorte initial. Les deux courbes ont des allures similaires : leur coefficient de corrélation est de 0,79 . L'effet cohorte apparaît donc très lié au contexte démographique du début de la carrière.

Ces résultats permettent d'interpréter l'effet de cohorte obtenu par l'estimation du modèle (1). Les cohortes 1979 à 1988 ont subi le choc du baby-boom et les effets d'un numerus clausus encore élevé. Elles ont des honoraires plus fai-

\section{Graphique XII \\ Interprétation des effets cohorte}

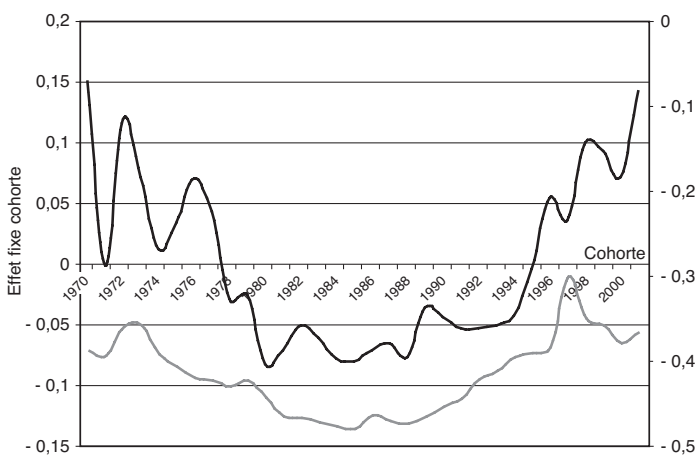

— Pas de tendance sur l'effet cohorte (sans indicatrice de participation)
Fonction linéaire quadratique de trois variables caractéristiques Fonction linéaire quadratique de trois variables caracter
de la démographie médicale à la date d'installation

Lecture : on rapproche les résultats obtenus grâce à deux types d'estimations :

- estimation du modèle (1) avec la contrainte identifiante « pas de tendance sur l'effet cohorte "

- remplacement de l'effet cohorte dans le modèle (1) par une fonction linéaire-quadratique de trois variables caractérisant la démographie médicale à la date d'installation.

Les deux courbes ont des allures similaires: le coefficient de corrélation entre les séries correspondant aux deux courbes est égal à 0,79 .

Champ : échantillon utilisé pour l'estimation : médecins du secteur 1, installés entre 1970 et 2001 et présents au moins une fois dans la base entre 1983 et 2004

Source: panel d'omnipraticiens libéraux du SNIR (CNAMTS), 1983-2004. bles que les cohortes installées au début des années 1970. L'impact de la diminution du numerus clausus intervenue à partir de 1978 se manifeste, neuf à dix ans plus tard, pour les cohortes installées à partir de la fin des années 1980. Celles-ci ont également profité d'une diminution des effectifs médicaux accélérée par le mécanisme d'incitation à la cessation d'activité (Mica). Toutes choses égales par ailleurs, leurs honoraires sont bien supérieurs à ceux des cohortes installées auparavant.

Comment expliquer le rôle décisif du contexte démographique au moment de l'installation? L'intensité de la concurrence entre médecins est pourtant déjà prise en compte dans le modèle par la densité d'omnipraticiens. Mais cette variable ne mesure pas l'intensité de la concurrence au moment de l'installation. Or cette circonstance apparaît cruciale. Lorsqu'il débute sa carrière, un médecin généraliste peut racheter la clientèle d'un médecin partant à la retraite ou constituer lui-même sa clientèle. Si beaucoup de débutants cherchent à s'installer en même temps dans une zone caractérisée par des départs en retraite peu nombreux, leur démarrage de carrière sera entravé par leur concurrence pour attirer des patients.

Nos résultats montrent que les modalités de début de carrière d'un médecin influencent ses honoraires de façon permanente. S'il ne parvient pas à constituer rapidement une clientèle suffisante, un médecin aura des revenus durablement plus faibles que ceux de ses collègues installés dans des conditions plus favorables. Pourquoi un démarrage insatisfaisant se transforme-t-il en handicap durable? Les explications restent à trouver. On peut penser que la taille de la clientèle d'un médecin non débutant influence la demande qui s'adresse à lui, dans la mesure où elle contribue à sa réputation. Une faible clientèle serait un mauvais signal pour un médecin non débutant: " ce médecin doit avoir un défaut, pour avoir si peu de patients »; «même s'il était compétent au départ, il a dû perdre la main $» . .$.

Ce scénario explicatif impose un retour sur le profil de carrière estimé par les effets fixes expérience. La concentration de l'activité sur les 12 premières années est peut-être un exercice imposé pour une profession libérale comme les médecins, qui doivent se constituer rapidement une clientèle et une réputation. Dans ce cas, leur profil de carrière ne révèlerait pas seulement une préférence en matière de répartition de l'effort de travail dans le cycle de vie, mais 
également l'obligation de ne pas rater son début de carrière (21).

\section{Une sélection accrue avec la diminution du numerus clausus}

L'évolution du numerus clausus introduit des taux de sélection variables en fin de première année des études de médecine. Cette sélection s'exerce sur les personnes inscrites en première année qui se présentent à un concours plus sélectif lorsque le numerus clausus diminue. Elle est renforcée en amont par un comportement d'auto-sélection: le nombre d'inscriptions en première année de médecine décroît aussi avec le numerus clausus (cf. graphique XIII).

L'effet de la sélection à l'entrée dans une profession a été étudié pour la Fonction publique française par Fougère et Pouget (2003). Les médecins ayant subi un taux de sélection plus sévère peuvent être en moyenne plus qualifiés pour l'exercice de la profession : on pourrait chercher à expliquer ainsi le relèvement de l'effet cohorte pour les médecins installés à partir du milieu des années 1990. Malheureusement, les données sur le nombre d'inscrits en première année de médecine ne sont pas disponibles pour les années antérieures à 1978 (et donc pour les cohortes installées avant 1987). Les données existantes sur cette question sont trop partielles pour permettre une analyse empirique complète.

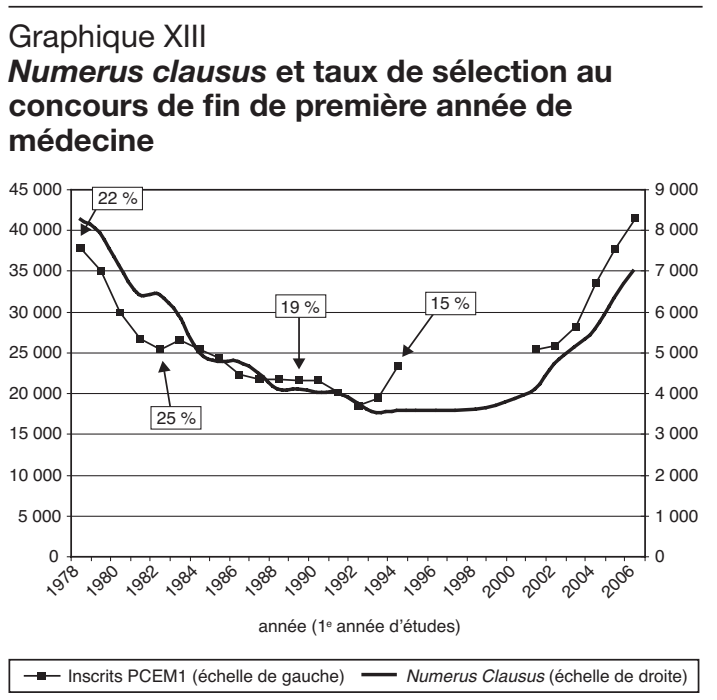

Lecture: le graphique représente la valeur du numerus clausus national et le nombre d'inscrits en première année de médecine. La rupture de la courbe est due au fait que l'information sur le nombre d'inscrits n'est pas disponible pour les années 1995 à 2000. Les étiquettes (par exemple $19 \%$ en 1989) donnent le pourcentage de reçus au concours de fin de première année de médecine.

Champ : étudiants ayant débuté leurs études de médecine entre 1978 et 2006

Source: panel d'omnipraticiens libéraux du SNIR (CNAMTS), 1983-2004 et données SISE (Système d'Information sur le Suivi de l'Etudiant) de la DEPP (Ministère de l'Education Nationale).

\section{Comparaison des distributions d'honoraires par cohorte}

$\mathbf{L}$ es différences d'honoraires entre cohortes sont-elles systématiques? L'analyse réalisée jusqu'à présent revient à comparer les honoraires moyens entre cohortes, en contrôlant par d'autres facteurs explicatifs. Or, l'hétérogénéité non expliquée par notre modèle est assez importante : le $\mathrm{R}^{2}$ s'élève à $27,55 \%$ seulement. Des différences interindividuelles entre praticiens sont possibles, qui permettraient à certains, plus dynamiques, de réussir malgré l'appartenance à une cohorte défavorable (22).

Une approche en termes de dominance stochastique permet de compléter l'analyse en considérant l'ensemble de la distribution des honoraires. Dans ce cadre, l'hétérogénéité non observée des médecins généralistes est intégrée à l'analyse et non négligée en tant que perturbation. Il s'agit ici d'examiner si le classement des cohortes obtenu précédemment est maintenu lorsque l'on considère les distributions.

\section{Critères de dominance stochastique}

Ces critères permettent de classer des distributions. Soit $F$ et $G$ les fonctions de répartition des honoraires pour les cohortes $C$ et $C^{\prime}$. Soit $x \geq 0$ un montant d'honoraires considéré. $F(x)$ et $G(x)$ définissent la proportion de médecins des cohortes $C$ et $C$ ' qui ont des honoraires inférieurs ou égaux à $x$. La distribution $F$ domine à l'ordre 1 la distribution $G$ si, quel que soit le montant $x$ d'honoraires considéré, la probabilité d'avoir un niveau d'honoraires supérieur à $x$ est plus importante avec $F$ qu'avec $G$.

On note $F \geq_{D S I} G$. Dans ce cas, la courbe représentative de la fonction de répartition $F(x)$ se situe toujours au-dessous de celle de $G(x)$.

Supposons que l'on propose à un médecin de choisir entre deux distributions, en situation d'ignorance a priori sur son niveau d'honoraires $x$. Tout individu dont l'utilité est fonction croissante des honoraires préfèrera la distribution

21. Il serait intéressant de pouvoir tester cette hypothèse avec des données concernant un type de profession libérale comme les taxis, dont l'activité ne dépend pas de la fidélisation d'une clientèle.

22. Par ailleurs, des effets de rattrapage sont envisageables, avec une hétérogénéité de l'effet de l'expérience selon les cohortes, qui permettrait aux médecins installés dans un contexte difficile de rattraper leur handicap au fil du temps. Cette question est étudiée à la fin de l'encadré 3. 
dominante $F$ à la distribution dominée $G$. Avec $F$ en effet, la probabilité d'être riche est supérieure, quel que soit le niveau de richesse considéré.

Très intuitif, le critère de dominance stochastique d'ordre 1 n'est pas toujours opératoire car les fonctions de répartition peuvent se croiser. Dans ce cas, on utilise le critère de dominance stochastique d'ordre 2, qui compare les montants de pauvreté moyens associés aux deux distributions. Pour chaque niveau d'honoraires $x$, on appelle montant de pauvreté moyen associé à la distribution $F$ le transfert moyen dont il faut faire bénéficier les individus d'honoraires inférieurs à $x$ pour les amener au niveau $x$. Si le montant de pauvreté moyen associé à la distribution $F$ est toujours inférieur au montant de pauvreté moyen associé à la distribution $G$, alors $F$ domine $G$ à l'ordre 2 .

Des procédures permettent de tester la dominance stochastique d'ordre 1 (notée DS1) ou 2 (DS2) et l'égalité des distributions. Nous adoptons la méthodologie suivie par Lefranc, Pistolesi et Trannoy (2004) et Pistolesi (2006).

\section{L'hétérogénéité individuelle ne compense pas le handicap d' une cohorte défavorable}

Nous avons tout d'abord considéré les fonctions de répartition des honoraires observés par cohorte. Pour clarifier l'analyse, nous nous focalisons sur cinq cohortes intéressantes : 1972, 1977, 1985, 1993 et 1999. Ces cohortes sont désignées par des étiquettes dans le graphique I. Les tests de dominance stochastique sont réalisés avec un risque de première espèce de $5 \%$. Ils conduisent à des résultats cohérents avec ceux obtenus dans l'analyse économétrique (cf. tableau 2) :

- la cohorte 1972 domine à l'ordre 1 les cohortes 1985,1993 et 1999 ;

- la cohorte 1985 est dominée à l'ordre 1 par les cohortes 1972 et 1999 et est égale à la cohorte 1993 ;

- la cohorte 1993 est dominée à l'ordre 1 par les cohortes 1972 et 1999 et est égale à la cohorte 1985 ;

- la cohorte 1999 est dominée à l'ordre 1 par la cohorte 1972 et à l'ordre 2 par la cohorte 1977. Elle domine à l'ordre 1 les cohortes 1985 et 1993.

Ces comparaisons sont toutefois d'une portée limitée car elles sont effectuées sur les distribu- tions d'honoraires bruts. Certes, l'hétérogénéité non observée des médecins est prise en compte. Mais les distributions d'honoraires sont ici comparées pour des dates et des niveaux d'expérience différents. Or les estimations des effets fixes ont montré l'importance de ces variables sur la détermination des honoraires.

Pour comparer les cohortes entre elles, il est plus pertinent d'éliminer de la variance des honoraires ce qui est dû aux autres facteurs que la cohorte et l'hétérogénéité non observée. L'estimation réalisée précédemment peut être exploitée pour combiner l'approche en termes de dominance stochastique avec une microsimulation. Nous pouvons simuler les honoraires qu'auraient perçus les médecins de l'échantillon s'ils avaient eu, hormis leur cohorte et leur hétérogénéité non observée, des caractéristiques semblables: la même expérience (10 ans), le même sexe (masculin), la même date d'observation (1995), des niveaux de densité correspondant aux moyennes observées, etc. (23). On obtient pour ces honoraires simulés des fonctions de répartition qui peuvent être comparées selon les critères de la dominance stochastique (cf. graphique XIV).

Le classement précédent est modifié par l'utilisation des honoraires simulés. Les différences

23. Plus exactement, les honoraires simulés sont définis à partir de l'estimation du modèle (1) de l'encadré 3 par: $\tilde{y}_{\text {ict }}=\hat{a}+\bar{D} \hat{b}+\bar{Z} \hat{d}+\hat{\eta}_{\text {IdF }}+\hat{\alpha}_{10}+\hat{\delta}_{1995}+\hat{\gamma}_{c}+\hat{\varepsilon}_{\text {ict }}$, où $\bar{D}$ correspond aux niveaux de densité moyens et où $\bar{Z}$ est le vecteur des indicatrices correspondant à un homme installé en lle de France comme libéral à temps plein, sans MEP.

Tableau 2

Tests de dominance stochastique entre les distributions par cohorte des honoraires simulés

\begin{tabular}{|c|c|c|c|c|c|}
\hline & 1972 & 1977 & 1985 & 1993 & 1999 \\
\hline 1972 & - & $>$ (SD1) & $>$ (SD1) & $>$ (SD1) & $=$ \\
\hline 1977 & - & - & $>$ (SD1) & $>$ (SD1) & $<$ (SD1) \\
\hline 1985 & - & - & - & $<$ (SD1) & $<$ (SD1) \\
\hline 1993 & - & - & - & - & $<$ (SD1) \\
\hline
\end{tabular}

Lecture: la distribution des honoraires simulés de la cohorte 1972 domine à l'ordre 1 (> (SD1)) la distribution des honoraires simulés de la cohorte 1977.

La distribution des honoraires simulés de la cohorte 1985 est dominée à l'ordre 1 (< (SD1)) par la distribution des honoraires simulés de la cohorte 1993.

La distribution des honoraires simulés de la cohorte 1972 est égale (=) à la distribution des honoraires simulés de la cohorte 1999 (hypothèse acceptée au seuil de $10 \%$ ).

Champ : échantillon utilisé pour l'estimation : médecins du secteur 1 installés entre 1970 et 2001 et présents au moins une fois dans la base entre 1983 et 2004

Source: panel d'omnipraticiens libéraux du SNIR (CNAMTS), 1983-2004. 
d'honoraires entre les cohortes sont plus marquées : toutes les cohortes peuvent maintenant être classées en utilisant le critère de dominance stochastique d'ordre 1. Les cohortes 1993 et 1999, qui étaient désavantagées par un faible niveau d'expérience observée, obtiennent un meilleur positionnement. La cohorte 1985 est maintenant dominée à l'ordre 1 par toutes les cohortes, et en particulier par 1993. Les distributions d'honoraires des cohortes 1972 et 1999 sont maintenant égales (au sens du test) : à caractéristiques observables identiques, les cohortes les plus récentes ont la même distribution d'honoraires que les cohortes anciennes les plus favorisées, ce qui confirme le redressement de la situation financière deté individuelle non observée ne permettent pas de compenser les différences considérables repérées, en moyenne, pour les cohortes cohortes les plus récentes.

$\mathrm{Au}$ total, l'analyse en termes de dominance stochastique confirme les conclusions de l'approche économétrique. Appartenir à une "mauvaise » cohorte est déterminant pour l'ensemble de la carrière d'un médecin généraliste ; les écarts liés à l'hétérogénéis.

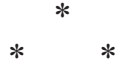

La régulation pratiquée en France ne permet pas d'effectuer une réelle étude de causalité : aucun échantillon témoin ne peut être défini car tous les étudiants de médecine d'une même génération sont soumis à la même régulation démographique. Toutefois, nous avons pu mettre en évidence l'importance du lien entre l'effet de la cohorte et la régulation de la démographie médicale. Les effets négatifs estimés pour les cohortes des années 1980, qui sont les plus nombreuses, amènent naturellement à s'interroger sur l'opportunité du relèvement actuel du numerus clausus. Les justifications généralement avancées se réfèrent à la diminution prévisible du nombre de médecins. Toutefois, la hausse du numerus clausus peut avoir un effet contraire à celui recherché, si elle contribue, par ses effets négatifs sur les revenus, à détériorer l'attractivité de l'exercice de la médecine générale.

Ces résultats révèlent l'existence d'une très grande disparité dans la situation des médecins généralistes selon leur cohorte d'appartenance. Certaines générations de médecins peuvent trouver leur compte dans le système actuel du paiement à l'acte, d'autres être plus ouvertes à d'autres modes de paiement. Les acteurs des négociations conventionnelles peuvent ainsi évoluer en fonction des situations relatives des différentes cohortes. La création en 1984 de MG France, premier syndicat de médecins généralistes, peut être mise en rapport avec la dégradation de la situation financière des cohortes installées dans les années 1980.

D'autres professions peuvent être affectées par les fluctuations démographiques et le babyboom. Létude des carrières salariales montre que les cohortes les plus nombreuses souffrent aussi d'un choc négatif sur les salaires initiaux (Welch,1979). Les estimations que nous avons obtenues pour les écarts entre cohortes de médecins sont similaires sur les honoraires et le revenu. Il est donc possible de se limiter aux honoraires pour les étudier. L'évaluation des revenus des médecins sera en revanche très utile, à l'avenir, pour comparer la situation financière et les profils de carrière des médecins avec ceux de salariés de niveau de formation équivalent.

\section{Graphique XIV \\ Fonctions de répartition des honoraires simulés pour cinq cohortes}

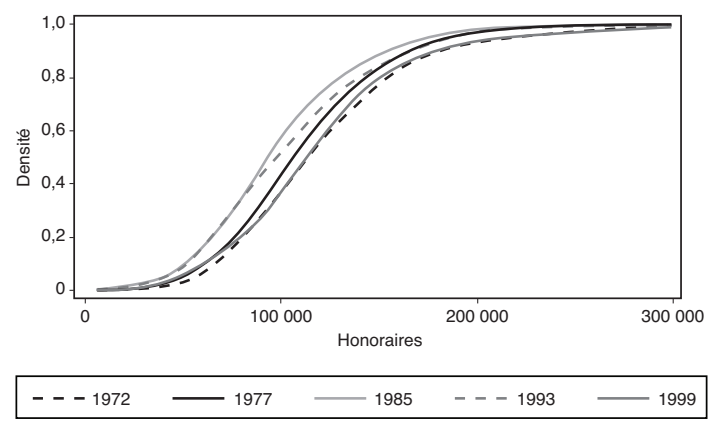

Lecture : il s'agit des fonctions de répartition des honoraires simulés. Ces honoraires simulés correspondent à ceux qu'auraient perçus les médecins de l'échantillon s'ils avaient eu, hormis leur cohorte et leur hétérogénéité non observée, des caractéristiques observables semblables (expérience: 10 ans, sexe: masculin, date d'observation : 1995, niveaux de densité correspondant aux moyennes observées, etc.)

Champ : échantillon utilisé pour l'estimation : médecins du secteur 1, installés entre 1970 et 2001 et présents au moins une fois dans la base entre 1993 et 2004.

Source: panel d'omnipraticiens libéraux du SNIR (CNAMTS), 1983-2004. 


\section{BIBLIOGRAPHIE}

Allain O. (1997), « La décomposition des évolutions de salaire selon l'âge, la cohorte et la période », Communication pour les $4^{\text {èmes }}$ journées d'étude sur les données longitudinales dans l'analyse du marché du travail (Céreq, Lasmas Idl, LES), Paris, 22-23 mai.

Aubert P. et Crépon B. (2003), « La productivité des salariés âgés : une tentative d'estimation», Économie et Statistiques, no 368.

Audric S. (2006), "Analyse des carrières des médecins libéraux à partir des données de panel », document de travail Drees, série statistiques, $\mathrm{n}^{\circ} 96$.

Bessière S., Breuil-Genier P. et Darriné S. (2004), «La démographie médicale à l'horizon 2025 : une actualisation des projections au niveau national », Études et Résultats $\mathrm{n}^{\circ} 352$, Drees.

Billaut A. (2006), "Les affectations en troisième cycle des études médicales en 2005, suite aux épreuves classantes nationales », Études et Résultats $\mathrm{n}^{\circ} 474$, Drees.

Bolduc D., Fortin B. et Fournier M.-A. (1996), "The Effect of Incentive Policies on the Practice Location of Doctors: A Multinomial Probit Analysis ", Journal of Labour Economics, vol. 14, pp. 703-732.

Bourdallé G. et Cases C. (1996), « Les taux d'activité des 25-60 ans: les effets de l'âge et de la génération ", Économie et Statistiques, n ${ }^{\circ} 300$, pp. 83-93.

Bourgueil Y. (2007), « La démographie médicale : constats, enjeux et perspectives $»$, Regards, $\mathrm{n}^{\circ} 31$, pp. 34-46.

Breuil-Genier P. (2003), « Honoraires et revenus des professions de santé en milieu rural ou urbain », Études et Résultats n ${ }^{\circ} 254$, Drees.

Cour des Comptes (2007), "Sécurité Sociale $2007 », \quad$ http ://www.ccomptes.fr/CC/documents/ RELFSS/07-securite-sociale.pdf

Deaton A. (1997), The Analysis of Household Surveys: A Microeconometric Approach to Development Policy, The Johns Hopkins University Press/World Bank : Baltimore.

Delattre E. et Dormont B. (2003), « Fixed Fees and Physician-Induced Demand: a Panel Data
Study on French Physicians », Health Economics, vol. 12, pp. 741-754.

Delattre E. et Dormont B. (2005), « La régulation de la médecine ambulatoire en France : quel effet sur le comportement des médecins libéraux ?», Solidarité Santé, nº 1, pp. 135-161, Drees.

Déplaude M.-O. (2007), «L'emprise des quotas. Les médecins, l'Etat et la régulation démographique du corps médical (année 1960-années 2000) », Thèse pour le doctorat en science politique, Université Paris 1-Panthéon Sorbonne.

ECOSANTE (2007), $h t t p: / / w w w . e c o s a n t e . f r /$

Fivaz C. et Le Laidier S. (2001), « Une semaine d'activité des généralistes libéraux », Cnamts, Point Stat n ${ }^{\circ} 33$.

Fougère D. et Pouget J. (2003), «Les déterminants économiques de l'entrée dans la fonction publique », Économie et Statistiques, no 369-370.

HCAAM - Haut conseil pour l'avenir de l'assurance maladie (2007), « Avis sur les conditions d'exercice et de revenu des médecins libéraux », 24 mai 2007, http ://www.sante.gouv.fr/htm/dossiers/hcaam/avis_240507.pdf

Heckman J. (1979), « Sample Selection Bias as a Specification Error », Econometrica, vol. 47, n 1 , pp. 153-161.

Kessler D. et Masson A. (1985), « Petit guide pour décomposer l'évolution d'un phénomène en termes d'effet d'âge, de cohorte et de moment », in Cycle de vie et générations, Kessler D., Masson A. et Strauss-Kahn D., Economica, Paris.

Kirby J. L. (2002), « The Health of Canadians The Federal Role » The Standing Senate Committee on Social Affairs, Science and Technology.

Koubi M. (2003a), « Les trajectoires professionnelles : une analyse par cohorte ", Économie et Statistiques, $\mathrm{n}^{\circ}$ 369-370.

Koubi M. (2003b), « Les carrières salariales par cohorte de 1967 à $2000 »$, Économie et Statistique, $\mathrm{n}^{\circ} 369-370$.

Lazear E. (1981), «Agency, Earnings Profiles, Productivity and Hours Restrictions ", The American Economic Review, vol. 71, pp. 606-620. 
Lazear E. and Moore R. (1984), «Incentives, Productivity and Labor Contracts », The Quarterly Journal of Economics, vol. 99, pp. 275-296.

Lefranc A., Pistolesi N. et Trannoy A. (2004), « Le revenu selon l'origine sociale », Économie et Statistiques, $\mathrm{n}^{\circ} 371$.

Legendre N. (2007), « Les revenus libéraux des médecins en 2004 et 2005 », Études et Résultats $\mathrm{n}^{\circ} 56$.

Lollivier S. et Payen F. (1990), « L'hétérogénéité des carrières individuelles mesurées sur données de panel », Économie et Prévision, n 92-93, pp. 87-95.

Lucas-Gabrielli V. et Sourty-Le Guellec M.-J. (2004), «Evolution de la carrière libérale des médecins généralistes selon leur date d'installation (1979-2001) », Questions d'Économie de la Santé, Irdes, $n^{\circ} 81$.

McGuire T. (2000), "Physician Agency » in A. Culyer et J. Newhouse, Handbook of health economics, vol. 1A, Elsevier Science, Amsterdam.

Mincer J. (1974), «Schooling, Experience and Earnings », New York : National Bureau of Economic Research.

OCDE (2006), « The Supply of Physician Services in OECD Countries », OECD Health Working Papers $n^{\circ} 21$, OECD Directorate for Employment, Labour and Social Affairs (DELSA/HEA/WD/ HWP(2006)(1).

ONDPS (2005), « Rapport annuel», www.sante. gouv.fr/ondps
Pistolesi N. (2006), «L'égalité des chances en France et aux États-Unis : le rôle de l'effort, des circonstances et de la responsabilité », Thèse pour le doctorat en science économique, Université de Cergy Pontoise.

Rizzo J. et Zeckhauser R. (2007), «Pushing Incomes to Reference Points: Why do Male Doctors Earn More?», Journal of Economic Behavior and Organization, vol. 63, $\mathrm{n}^{\circ} 3$, pp. 514-536.

Samson A.-L. (2006), « La dispersion des honoraires des omnipraticiens », Études et Résultats $n^{\circ} 482$, Drees.

Sénat (2007), « Offre de soins : comment réduire la fracture territoriale ? » Rapport d'information $\mathrm{n}^{\circ} 14$ de Jean-Marc Juilhard, sénateur du Puyde-Dôme (http ://www.senat.fr/rap/r07-014/r070141.pdf)

URML Ile de France (2007), « L'épuisement professionnel des médecins libéraux : témoignages, analyses et perspectives », dossier de presse, 27 juin 2007.

Verbeek M. et Nijman T. (1992), «Testing for Selectivity Bias in Panel data Models ", International Economic Review, Vol. 33, n 3, 681-703.

Vanderschelden, M. (2007), «Les affectations des étudiants en médecine à l'issue des épreuves classantes nationales en 2006 ", Études et Résultats $\mathrm{n}^{\circ} 571$, Drees.

Welch F. (1979), «Effects of Cohort Size on Earnings: the Baby-boom Babies' Financial Bust », Journal of Political Economy, vol. 87, $\mathrm{n}^{\circ} 5$, pp. S65-S97. 